\title{
Prediction of Molecular Interactions And Physicochemical Properties Relevant For Vasopressin V2 Receptor Antagonism
}

Ania de la Nuez Veulens ( $\boldsymbol{\sim}$ ania.delanuez@fbio.uh.cu )

University of Havana: Universidad de la Habana https://orcid.org/0000-0002-0478-1890

Yoanna María Álvarez Ginarte

University of Havana: Universidad de la Habana

Rolando Eduardo Rodríguez Fernandez

DNA digest Inc

Fabrice Leclerc

Université Paris-Sud: Universite Paris-Saclay

Luis Alberto Montero Cabrera

University of Havana: Universidad de la Habana

\section{Research Article}

Keywords: V2R, AVP, Vasopressin Antagonist, GPCR, QSAR, Docking, Molecular Dynamics Simulations

Posted Date: July 26th, 2021

DOI: https://doi.org/10.21203/rs.3.rs-639693/v1

License: (9) This work is licensed under a Creative Commons Attribution 4.0 International License. Read Full License 


\section{Abstract}

We have developed two ligand and receptor-based computational approaches to study the physicochemical properties relevant to the biological activity of vasopressin V2 receptor (V2R) antagonist and eventually to predict the expected binding mode to V2R. The obtained Quantitative Structure Activity Relationship (QSAR) model showed a correlation of the antagonist activity with the hydration energy $\left(\mathrm{EH}_{2} \mathrm{O}\right)$, the polarizability $(\mathrm{P})$ and the calculated partial charge on atom N7 (q6) of the common substructure. The first two descriptors showed a positive contribution to antagonist activity, while the third one had a negative contribution. V2R was modeled and further relaxed on a 1-palmitoyl-2-oleoyl-snglycero-3-phosphocoline (POPC) membrane by molecular dynamics simulations. The receptor antagonist complexes were guessed by molecular docking, and the stability of the most relevant structures were also evaluated by molecular dynamics simulations. As a result, amino acid residues Q96, W99, F105, K116, F178, A194, F307, and M311 were identified with the probably most relevant antagonist-receptor interactions on the studied complexes. The proposed QSAR model could explain the molecular properties relevant to the antagonist activity. The contributions to the antagonist-receptor interaction appeared also in agreement with the binding mode of the complexes obtained by molecular docking and Molecular Dynamics. These models will be used in further studies to look for new V2R potential antagonist molecules.

\section{Introduction}

Autosomal Dominant Polycystic Kidney Disease (ADPKD) is a genetic condition with an incidence of 1:400, to $1: 1000$ in the world population. Patients develop multiple fluid-filled cyst in both kidneys, increasing the total kidney volume and leading to Chronic Kidney Disease. In contrast to the normal renal cells, the PKD cystic cells have an increased cAMP induced proliferation by activating the Ras/BRaf/MEK/ERK pathway, mediated by the decrease in intracellular calcium levels [1]. The absence of renal cysts in PCK rats lacking arginine vasopressin (AVP) might indicate that the receptors on the collecting ducts activating other Adenylate Cyclases, do not have a significant role on the generation of cysts $[2,3]$.

AVP is a nonapeptide hormone consisting of a six amino acid ring closed by a disulphide bridge between cysteines 1 and 6 , followed by a tripeptide tail. This hormone is synthesized in the hypothalamus, principally produced by neurons with the cell body within the supraoptic and the paraventricular nuclei, and their axon terminations in the neural lobe of the posterior pituitary gland in which AVP is released into the circulation [4]. The primary function of AVP is to maintain body fluid balance by keeping plasma osmolality within narrow limits [5]. Increase in plasma osmolality or decrease in plasma volume trigger its release to induce expression of water transport proteins in the late distal tubule and collecting ducts of the kidneys, to increase water reabsorption [5]. Due to its role in the regulation of osmolarity by increasing the ability of the kidney to reabsorb water reducing the urinary volume, it is also known as Antidiuretic Hormone (ADH). 
AVP physiological roles are mediated by three receptor subtypes V1a, V1b (also called V3), and V2 all belong to vasopressin/oxytocin receptor family, and they are class-A G-protein coupled receptor (GPCR). The $\mathrm{V} 1$ a receptors are mainly distributed on vascular smooth muscle, but also present in myocardium, platelets, and hepatocytes. V1a stimulation is associated with vasoconstriction and cardiac hypertrophy, together with platelet aggregation, and glycogenolysis $[4,6,7]$. The $\mathrm{V} 1 \mathrm{~b}$ receptors have little selective distribution, and their activation is part of the adaptive reaction to stress, leading to stimulation of adrenocorticotropic hormone and endorphin release[4,6]. The activity of each receptor is mediated by $G$ proteins which activate a phosphatidyl-inositol-calcium second messenger system.

The $\mathrm{V} 2$ receptor $(\mathrm{V} 2 \mathrm{R})$ is expressed predominantly in the principal cells of the renal collecting duct system, in which its activation leads to increased resorption of free water [4-6]. V2R is the major activator of adenylyl cyclase signaling pathway in principals cell of collecting ducts in kidney. The increase of cAMP intracellular concentration by the activation of V2R promote proliferation in PKD cystic cells, suggesting that V2R antagonists can be used as treatment for PKD to retard development and growth of the cysts [8-10].

Selective peptide antagonist of V2R were developed $[11,12]$ but these efforts have encountered many obstacles due to the residual agonist activity, heterogeneity in species response and very low oral bioavailability limiting their clinical use [13]. These limitations make the development of new non-peptide $\mathrm{V} 2 \mathrm{R}$ antagonists more attractive.

Orally and intravenously active non-peptide vasopressin receptor antagonists are called vaptans. The first success in this field was mozavaptan (OPC-31260), a benzazepine derivative and a potent, selective, competitive and orally active vasopressin V2 receptor antagonist [14], soon followed by its use in humans [15] and the first to gain approval for clinical use in Japan since 2006 for the treatment of tumorassociated to Syndrome of inappropriate Antidiuretic Hormone secretion (SIADH).

Among the non-peptide V2R antagonists developed and experimentally tested, only two compounds of this class have been approved in the United States, Canada and the European Union [13]. The U.S. Food and Drug Administration (FDA) approved conivaptan and tolvaptan for euvolemic and hypervolemic hyponatremia [16]. Tolvaptan is also approved to slow kidney function decline in adults at risk of rapidly progressing Autosomal Dominant Polycystic Kidney Disease (ADPKD), the only drug approved to treat this condition so far [16].

Besides the retardation of progressive renal failure in ADPKD and the treatment for euvolemic or hypervolemic hyponatremia, experiments show that $\mathrm{V} 2 \mathrm{R}$ antagonists can be used for rescue treatment in Congenital Nephrogenic Diabetes Insipidus [17], treatment of diabetic nephropathy [18], congestive heart failure [19], and also in the prevention of ascites formation in cirrhosis [20]. Other indications for treatment with vasopressin-receptor antagonists will probably emerge.

In this work we have used a computational modeling approach to study a family of non-peptide V2R antagonists, with their $\mathrm{IC}_{50}$ being determined experimentally, identifying physicochemical properties 
relevant to the biological activity of these compounds and relevant interactions with V2R.

\section{Methods}

\subsection{Data set of V2R Antagonist}

To obtain a reliable QSAR model we used chemical information from the assays AID-217680, AID2176881 and AID-483985, all in the PubChem's Bio Assay Database (https://pubchem.ncbi.nlm.nih.gov) completing a series of 53 antagonists. In these assays, the biological activity at V2R was assessed as the displacement of [3H]-AVP from its AVP-V2R binding site and the inhibition of intracellular cAMP accumulation. The $\mathrm{IC}_{50}$ value is the concentration of compound which inhibits [3H]-AVP binding by $50 \%$. In our study, the negative logarithm of the biological activity, $\mathrm{pIC}_{50}$, was used as the dependent variable to determine QSAR correlation equations. The 3D structure of each antagonist was generated from its SMILES in Pubchem database.

\subsection{Estimation of molecular properties}

The specific action of drugs depend on many intrinsic features such as hydrophobic, electronic and steric properties. In a QSAR model the biological activity is expressed as a function of molecular descriptors. A molecular descriptor encode as a number, the result of a mathematical and logical procedure using the information of specific properties of molecules. In this study we calculated as hydrophobic descriptors, the logarithm of the octanol/water partition coefficient (LogP) and hydration energy; as steric descriptors: approximate surface area (ASA), grid surface area (GSA), molar volume (MV)[21, 22], and molar refractivity (MR); as for electronic descriptors: polarizability (P) [23], dipole moment ( $\mu$ ), total energy (TE), highest occupied molecular orbital eigenvalue (eHOMO), lowest unoccupied molecular orbital eigenvalue (eLUMO), partial atomic charges of the pharmacophore atoms (q1 to q11), electrophilicity index ( $\omega$ ), chemical hardness ( $\eta$ ), chemical softness (s) [24]. Electronic descriptors were calculated by Kohn-Sham's DFT B3LYP/6-31G method as included in Gaussian 09 program routines [25]. The other descriptors were calculated with QSAR propierties available in Hyperchem v8 software [26].

\subsection{Cluster analysis}

Cluster analysis is used in QSAR models to build the training and test sets as well as to determine the structural diversity of the dataset. In cluster analysis, the antagonists were classified in groups, called clusters, with a relative homogeneity. The structural diversity or similarity between the compounds is determined by calculating the Euclidean distance between each couple of objects: the smaller the distance, the more of the objects are considered similar to each other [27]. To check the structural diversity of the dataset and to define the number of possibles clusters, a hierarchical cluster analysis of these molecules was performed using k-NNCA algorithm to construct the dendrogram. The complete linkage distance (Euclidean metric) was used as the connection function to merge the objects into clusters. The complete linkage measures the proximity between two groups, calculating the distance among the farthest objects, or the similarity among the objects with lesser similarities. The Euclidean 
distance is the square root of the sum of the squared differences among the values of two objects for each variable $[28,29]$.

To select the training and test sets we used the k-mean cluster algorithm (k-MCA). Such algorithms use a switching method to divide $\mathrm{N}$ data points into $\mathrm{k}$ groups (clusters) to minimize the sum of distances/dissimilarities among the objects within the same cluster. The k-mean approach require that $\mathrm{k}$ (the number of clusters) must be known before clustering [29]. The k values were set taking into account the dendrogram obtained for the first cluster analysis. Both hierarchical and partitional (non-hierarchical) cluster analyses were implemented using the STATISTICA 8 software [30]. After the cluster analysis, the compounds were separated in two sets: $80 \%$ of compounds in each cluster were selected for the training set and $20 \%$ of each cluster for the test set. The training set was used to develop the QSAR model and the test set was used for external cross-validation of the model.

\subsection{QSAR model}

A correlation matrix was performed to determine among the calculated molecular descriptors the ones that do not correlate to each other. A Genetic Algorithm (GA) was used as a metaheuristic method for the molecular descriptors selection and optimization of the functions [31-34]. The length of the equation was set for three terms and a constant, and the GA was used for input selection to establish which of the descriptors will have the best multiple linear regression (MLR). Several statistical parameters were employed to validate the model. A good QSAR model should have the highest squared correlation coefficient, $\mathrm{R}^{2}$ and Fisher-test, with the lowest standard deviation (S). The P-value is another important parameter used for modeling validation and it should be lower than 0.01 . The predictive power of the model was then determined by examining the leave-one-out (LOO) cross-validation $\left(q^{2}\right)$. The $q^{2}$ is known as the predictive variance, with a value higher than 0.5. To validate the QSAR model, an external prediction test set of compounds (in the model range) was used, as the predictive ability of a QSAR model shall only be estimated using an external test set [35,36]. All the procedures used to build the QSAR model were performed with BuildQSAR software [37] and validated with STATISTICA 8 software [30].

\subsection{The applicability domain}

The applicability domain is the theoretical region of the chemical space, defined by the model descriptors and the modeled response, therefore by the nature of the compounds in the training and test sets, as represented in each model by specific molecular descriptors. The applicability domain of a QSAR model is "the range within which it tolerates a new molecule" [28], a QSAR model is only valid within the same domain for which it was developed. Even if the models are developed for the same chemical structures, the applicability domain for new structures can differ from model to model, depending on specific descriptors.

In multiple predictor models, performing simple single-variable range checks is not sufficient to verify the applicability domain. For MLR, one of the most used approaches with normally distributed data for a multiple predictor problem is a distance-based measure like the leverage (h). As the leverage of a 
compound measures its influence on the model, it becomes possible to verify whether a new chemical will fit within the structural model domain. The leverage used as a quantitative measure of model applicability domain is also suitable for evaluating the degree of extrapolation, which represents a sort of compound distance from the model experimental space. The warning leverage $\left(h^{\star}\right)$ is a critical value or cut-off. Predictions should be considered unreliable for compounds with high leverage $(h>h \star$, being the critical value $h^{\star}=3 p^{\prime} / n$, where $p^{\prime}$ is the number of model variables plus one, and $n$ is the number of the compounds) [28].

\subsection{V2R modeling}

GPCRdb template tool (https://gpcrdb.org/structure/template_selection) was used to identify the possible templates for V2R and the human OX2 orexin receptor (PDB ID 4SOV) was selected for V2R modeling. The alignment between the template sequence and V2R was made with a Clustal $X$ v2.1 profile [38] considering the alignment already obtained with the GPCRdb template tool. The model was built by homology with YASARA program v12.8.26 [39]. YASARA uses knowledge-based energies to validate the receptor model normalizing them to remove the dependencies on the size and shape of the protein, and on its amino acid composition, obtaining estimates for the expected average energy and its standard deviation from gold standard reference structures. Then it calculates how many standard deviations it is away from the average, thereby obtaining a Z-score to evaluate the quality of the models.

To minimize and equilibrate the receptor model it was inserted in a 1-palmitoyl-2-oleoyl-sn-glycero-3phosphocholine (POPC) membrane patch, generated with the VMD [40] Build Membrane plugin, which mimics its natural environment. POPC was selected because its abundance in biological membranes and because it does not introduce any curvature in the structure. The receptor model was oriented on the membrane, according to the orientation of the orexin receptor (ID PDB 4SOV) on the membrane in the MemProtMD database [41]. The membrane with the receptor inserted was oriented in the XY plane. The system was solvated in a $93 \times 92 \times 113 \AA$ periodic box of TIP3P water and $\mathrm{NaCl}$ was added at physiological concentration, neutralizing the system. The energy of the system was minimized with 1000 steps of conjugate gradient and a further equilibration for $10 \mathrm{~ns}$ ( $0.5 \mathrm{~ns}$ to $300 \mathrm{~K}$ lipids tails; $0.5 \mathrm{~ns}$ to 300 $\mathrm{K}$ membrane; $9 \mathrm{~ns}$ to $310 \mathrm{~K}$, on the whole system) was performed with NAMD2 [42] with an integration time step of $2 \mathrm{fs}$. A Langevin thermostat and barostat were used to maintain an NPT system, the cut-off for non-bonding interactions was $12 \AA$, a smooth switching function at $10 \AA$ was used for van der Waals interactions, and Particle Mesh Ewald (PME) for electrostatics interactions. The membrane parameters were checked with MEMBPLUGIN [43] of VMD.

\subsection{Molecular docking}

The receptor model obtained in the previous simulation was used to perform docking studies to predict the binding modes of three antagonist of the studied family. The receptor model and the antagonists were prepared using AutoDockTools [44] to perform the molecular docking with AutoDock Vina [45], where the antagonist and the sidechains of the receptor residues (Q96, W99, F105, K116, and F307) were flexible. The search space was restricted to a $28 \times 20 \times 14 \AA$ box. The default parameters for 
configuration files were used in Autodock Vina, running it 5 times and saving 10 conformations of each compound for each run to generate a total of 50 conformations for each compound. The docking results were visually analyzed using UCSF Chimera [46]. All docking results were clustered using a tolerance value of $2.0 \AA$ RMSD and three representative orientations in the binding site were identified to select one conformation per compound. The interactions between the selected antagonist conformation and the V2R were analyzed using BINANA [47] and OpenEye Scientific Software [48]

\subsection{Molecular dynamic simulations of complexes in POPC}

The topologies and parameters of the selected antagonists for CHARMM 36 force field [49] were generated using CHARMM-GUI $[50,51]$. The complex in the POPC membrane was minimized $(10000$ steps) and equilibrated for $100 \mathrm{ps}$ at $310 \mathrm{~K}$ with NAMD2. A molecular dynamic simulation was performed for $50 \mathrm{~ns}$ ( $310 \mathrm{~K}$, NTP and constant area) with NAMD2, saving frames and calculating energy every 5000 steps.

For the equilibration and the production simulations, a Langevin thermostat and barostat were used to maintain an NPT system, the cut-off for non-bonding interactions was $12 \AA$, a smooth switching function at $10 \AA$ was used for van der Waals interactions and Particle Mesh Ewald (PME) for electrostatics interactions. The integration time step was $2 \mathrm{fs}$. VMD [40] was used for the analysis and visualization of the molecular dynamic simulations.

\subsection{Complex free energy calculations using linear interaction energy methods}

The linear interaction energy (LIE) method was used to estimate the free energy of antagonist-receptor binding. For this purpose, in addition to the previous simulation of the complex in the membrane in a cubic water box, a second simulation of the antagonist only in the water box is needed, which was carried out using the same parameters as the simulation of the antagonist-receptor complexes. Eq. 1 shows the improved LIE formula suggested by Almlöf et al. $[52,53]$ taking into account the intra-ligand electrostatic interactions.

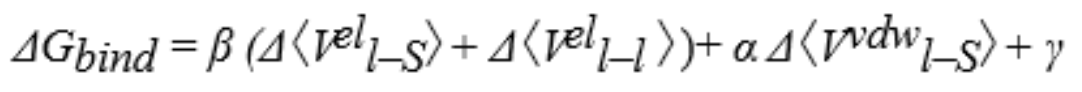

Where $\llbracket V e^{l} /$-Sland $\square W^{W d w} /$-Slare MD-generated interaction energy averages from the non-bonded electrostatic and van der Waals interactions of the ligand with its surrounding environment (s). $\square V^{e l} /-\mathbb{Q}$ is the electrostatic intramolecular ligand-ligand average energy. The $\Delta$ 's denote the change in average values when transferring the ligand from solution (free state) into the binding site of the solvated receptor (bound state). Coefficients $a$ and $\beta$ are scaling factors for the energy terms, while $\gamma$ is an empirical constant. In this study, $a$ was considered as 0.18 which is deemed as a robust value from previous works [52-54]. The $\beta$ specific values for each antagonist was calculated using the parameterization model $\mathrm{E}$ proposed by Almlöf et al. [52, 53] (Eq. 2). 


$$
\beta_{F E P}=\beta_{0}+\frac{\Sigma_{i} w_{i} \Delta \beta_{i}}{\Sigma_{i} w_{i}}
$$

Where $w_{i} \beta_{0}$, and $\Delta \beta_{i}$, were calculated from explicit solvent FEP calculations of single chemical group ( $w_{i}$ $=1$ if group is neutral and 11 if it is an anion or a cation), $\beta_{0}=0.43$ and $\Delta \beta_{i}$ was obtained by the model proposed by Almlöf et al[52].

The balance (difference) between the electrostatic (polar) and the van der Waals (nonpolar) contributions to the free energy binding in the LIE method was defined as the parameter $\mathrm{D}$ (Eq. 3)

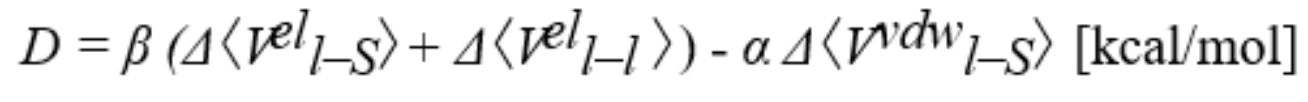

LIE-D is an approach based on the linear correlation between the $y$ coefficient and the $D$ parameter that accounts for the balance (difference) between the polar and nonpolar binding free energy contribution. The relationship between the $y$ coefficient and $D$ parameter takes the form:

$Y=f x D+g[\mathrm{kcal} / \mathrm{mol}](4)$

The values of $f$ and $g$ were estimated by Miranda et al [53] as -0.95 and -2.06 respectively.

\section{Results And Discussion}

\subsection{Construction of training and test sets using Cluster Analysis}

We selected a series of 53 compound antagonists of V2R to construct the training and test sets. All the selected molecules have the same core substructure Fig. 1, but show structure variability due to substituent structural diversity. All compounds have a common substructure (4-formamido-benzamide) remarked in a rectangle in the top panel of Fig. 1. The nitrogen of the benzamide in the common substructure (represented the partial charge q1) is part of a benzazepine, benzene-piperidine, or benzoxazine condensed ring. The R1 substituent is generally a ring except for compounds A03 and A07. We display in Table 1 the compounds we used in this study with PubChem ID and the experimental biological activities of $\mathrm{V} 2 \mathrm{R}$ antagonists' activity expressed as $\mathrm{IC}_{50}$ and $\mathrm{pIC}_{50}$, and the Supplementary Information Table S1 shows the values of all the calculated molecular descriptors.

Table 1: The experimental biological activities of V2R antagonists 


\begin{tabular}{|llll|}
\hline ID & PUBCHEM_CID & IC $_{50}(\mathrm{nM})$ & pIC $_{50}$ \\
\hline A01 & 119369 & 12 & 7.92 \\
\hline A02 & 151171 & 11 & 7.96 \\
\hline A03 & 977621 & 7100 & 5.15 \\
\hline A04 & 2981363 & 420 & 6.38 \\
\hline A05 & 2981862 & 6500 & 5.19 \\
\hline A06 & 2984025 & 6400 & 5.19 \\
\hline A07 & 5099582 & 8100 & 5.09 \\
\hline A08 & 10499401 & 1000 & 6.00 \\
\hline A09 & 10501216 & 760 & 6.12 \\
\hline A10 & 10524202 & 1800 & 5.74 \\
\hline A11 & 10527129 & 150 & 6.82 \\
\hline A12 & 10527137 & 1000 & 6.00 \\
\hline A13 & 10548204 & 400 & 6.40 \\
\hline A14 & 10548205 & 790 & 6.10 \\
\hline A15 & 10548464 & 200 & 6.70 \\
\hline A16 & 10550481 & 190 & 6.72 \\
\hline A17 & 10574499 & 14 & 7.85 \\
\hline A18 & 10595449 & 4100 & 5.39 \\
\hline A19 & 10598596 & 29 & 7.54 \\
\hline A20 & 10599369 & 77 & 7.11 \\
\hline A21 & 10599903 & 96 & 7.02 \\
\hline A22 & 10619160 & 680 & 6.17 \\
\hline A23 & 10620180 & 110 & 6.96 \\
\hline A24 & 10621059 & 530 & 6.28 \\
\hline A25 & 10622282 & 250 & 6.60 \\
\hline A26 & 10642000 & 980 & 6.01 \\
\hline A27 & 10647295 & 25 & 7.60 \\
\hline A28 & 10666852 & 200 & 6.70 \\
\hline
\end{tabular}




\begin{tabular}{|llll|}
\hline A29 & 10667727 & 210 & 6.68 \\
\hline A30 & 10668163 & 1900 & 5.72 \\
\hline A31 & 10690528 & 1400 & 5.85 \\
\hline A32 & 10692266 & 28 & 7.55 \\
\hline A33 & 10693776 & 44 & 7.36 \\
\hline A34 & 10713341 & 7600 & 5.12 \\
\hline A35 & 10716675 & 27 & 7.57 \\
\hline A36 & 10716676 & 24 & 7.62 \\
\hline A37 & 10741034 & 82 & 7.09 \\
\hline A38 & 10742994 & 13 & 7.89 \\
\hline A39 & 10743970 & 29 & 7.54 \\
\hline A40 & 10762667 & 400 & 6.40 \\
\hline A41 & 10762739 & 300 & 6.52 \\
\hline A42 & 10765617 & 50 & 7.30 \\
\hline A43 & 10766187 & 22 & 7.66 \\
\hline A44 & 10789935 & 58 & 7.24 \\
\hline A45 & 10810069 & 1200 & 5.92 \\
\hline A46 & 10810133 & 180 & 6.74 \\
\hline A47 & 10832492 & 1700 & 5.77 \\
\hline A48 & 10834036 & 2100 & 5.68 \\
\hline A49 & 10834761 & 170 & 6.77 \\
\hline A50 & 10837161 & 27 & 7.57 \\
\hline A51 & 10838492 & 20 & 7.70 \\
\hline A52 & 10838493 & 13 & 7.89 \\
\hline A53 & 11798122 & 71 & 7.15 \\
\hline
\end{tabular}

To classify the molecules of the datasets, depending on their structural variability we performed a hierarchical cluster analysis, the resulting dendrogram was constructed using the Euclidean distance ( $x$ axis) and the complete linkage (y-axis), illustrating the results of the k-NNCA developed in this dataset. The dendrogram shows 6 different subsets demonstrating the molecular variability among the 
compounds of this dataset (Fig. 2). To evaluate the output dendrogram and to split the whole dataset into training and test sets, we performed a k-mean cluster analysis (k-MCA) [55].

The selection of the training and test sets was carried out by randomly taking molecules belonging to each cluster. From the initial 53 compounds, 42 ( $80 \%$ of the dataset) were chosen to form the training set and the remaining 11 compounds, ( $20 \%$ of the dataset) were used as a test set for the external crossvalidation of the model.

\subsection{Development and validation of the QSAR model}

GA combined with MLR is widely used for QSAR and QSPR studies [31-34]. In this method, a GA is performed to search the feature space and select the major descriptors relevant to the activities or properties of the compounds. This method can deal efficiently with a large search space, and it has fewer chances to only find a local optimal solution than other algorithms. GA is a well-estimated method for parameter selection and to overcome the shortages of MLR in variable selection. After a GA, the MLR is employed to correlate the selected descriptors with the activity values using a classic regression method to yield the explicit equations.

The variables selected by the genetic algorithm as the best model of V2R antagonist activity are shown in Eq. 5. To further validate the variables thus obtained, we performed an MLR analysis of the 43 compounds on the initial training set, with the 11 compound test set for the external cross-validation.

pIC50 $=-7.968( \pm 3.584) \mathbf{q 6}+0.095( \pm 0.059) \mathrm{EH}_{2} \mathrm{O}+0.161( \pm 0.027) \mathrm{P}-5.842( \pm 2.894)(5)$

$n=43 ; R=0.89 ; R^{2}=0.80 ; s=0.40 ; F=53,61 ; p<0.0001 ; q^{2}=0.75$

\section{Test set:}

$n=11 ; R=0.86 ; R^{2}=0.74 ; s=0.41 ; F=25.04 ; p<0.0007 ; q^{2}=0.56$

The $\mathrm{R}^{2}$ (R-square statistic or coefficient of determination) indicate that the model could explain $80 \%$ of the variance for the experimental values of $\mathrm{plC}_{50}$. The model shows a $\mathrm{q}^{2}$ of 0.75 . This value of more than 0.5 could be considered as proof of the high predictive ability of the model, along with the good prediction of the test set $\left(R^{2}=0.74\right)$. The good $R^{2}$ and $q^{2}$ values obtained in Eq. 5 for both training and test set can be explained with the experimental values for all the compounds of the series. The calculated values for pIC50, are highly similar to the experimental, sustaining the reliability of the QSAR model (Fig. 3, Table 2). 
Table 2

Experimental and calculated values for the $\mathrm{pIC}_{50}$ of the dataset

\begin{tabular}{|c|c|c|c|}
\hline$I^{a}$ & $Y(o b s)^{b}$ & $Y(\text { calc })^{c}$ & Residuald $^{d}$ \\
\hline A01 & 7.92 & 7.41 & 0.51 \\
\hline $\mathrm{A} 02$ & 7.96 & 8.15 & -0.19 \\
\hline A03 & 5.15 & 5.37 & -0.22 \\
\hline $\mathrm{A} 04$ & 6.38 & 6.07 & 0.31 \\
\hline A05 & 5.19 & 5.60 & -0.41 \\
\hline A06 & 5.19 & 5.33 & -0.13 \\
\hline $\mathrm{A} 07^{*}$ & 5.09 & 5.39 & -0.29 \\
\hline $\mathrm{A} 08$ & 6.00 & 6.11 & -0.11 \\
\hline A09 & 6.12 & 5.66 & 0.46 \\
\hline A10* & 5.74 & 6.19 & -0.44 \\
\hline A11 & 6.82 & 7.17 & -0.35 \\
\hline A12 & 6.00 & 6.25 & -0.25 \\
\hline A13 & 6.40 & 6.16 & 0.24 \\
\hline A14 & 6.10 & 6.13 & -0.03 \\
\hline A15* & 6.70 & 6.24 & 0.46 \\
\hline A16 & 6.72 & 6.96 & -0.24 \\
\hline A16* & 6.72 & 6.96 & -0.24 \\
\hline A17 & 7.85 & 7.51 & 0.34 \\
\hline A18 & 5.39 & 5.79 & -0.41 \\
\hline A19 & 7.54 & 7.29 & 0.25 \\
\hline A20* & 7.11 & 7.34 & -0.23 \\
\hline A21 & 7.02 & 7.15 & -0.14 \\
\hline \multicolumn{4}{|c|}{${ }^{a}$ ID of compounds in the study. Chemicals marked with an asterisk in the test set } \\
\hline \multicolumn{4}{|c|}{${ }^{b}$ Experimental values of the effective dose } \\
\hline \multicolumn{4}{|c|}{${ }^{\mathrm{C}}$ Values calculated by Eq. 5} \\
\hline d Obse & us calcula & & \\
\hline
\end{tabular}




\begin{tabular}{|c|c|c|c|}
\hline$I^{a}$ & $Y(o b s)^{b}$ & $Y(\text { calc })^{c}$ & Residual $^{d}$ \\
\hline A22 & 6.17 & 6.31 & -0.15 \\
\hline A23 & 6.96 & 6.18 & 0.78 \\
\hline A24* & 6.28 & 5.52 & 0.76 \\
\hline A25 & 6.60 & 6.41 & 0.19 \\
\hline A26 & 6.01 & 5.90 & 0.11 \\
\hline A27 & 7.60 & 7.23 & 0.38 \\
\hline A28* & 6.70 & 6.21 & 0.49 \\
\hline A29 & 6.68 & 6.63 & 0.05 \\
\hline A30 & 5.72 & 6.25 & -0.53 \\
\hline A31 & 5.85 & 6.32 & -0.47 \\
\hline A32 & 7.55 & 6.87 & 0.68 \\
\hline A33 & 7.36 & 7.52 & -0.17 \\
\hline A34 & 5.12 & 5.63 & -0.52 \\
\hline A35 & 7.57 & 7.10 & 0.47 \\
\hline A36 & 7.62 & 7.30 & 0.33 \\
\hline A37 & 7.09 & 6.42 & 0.67 \\
\hline A38 & 7.89 & 8.19 & -0.30 \\
\hline A39 & 7.54 & 8.20 & -0.66 \\
\hline A40 & 6.40 & 6.41 & -0.01 \\
\hline A41* & 6.52 & 6.18 & 0.35 \\
\hline A42* & 7.30 & 7.64 & -0.33 \\
\hline A43 & 7.66 & 8.08 & -0.42 \\
\hline A44 & 7.24 & 7.06 & 0.18 \\
\hline
\end{tabular}

${ }^{a}$ ID of compounds in the study. Chemicals marked with an asterisk in the test set

${ }^{b}$ Experimental values of the effective dose

c Values calculated by Eq. 5

d Observed minus calculated values 


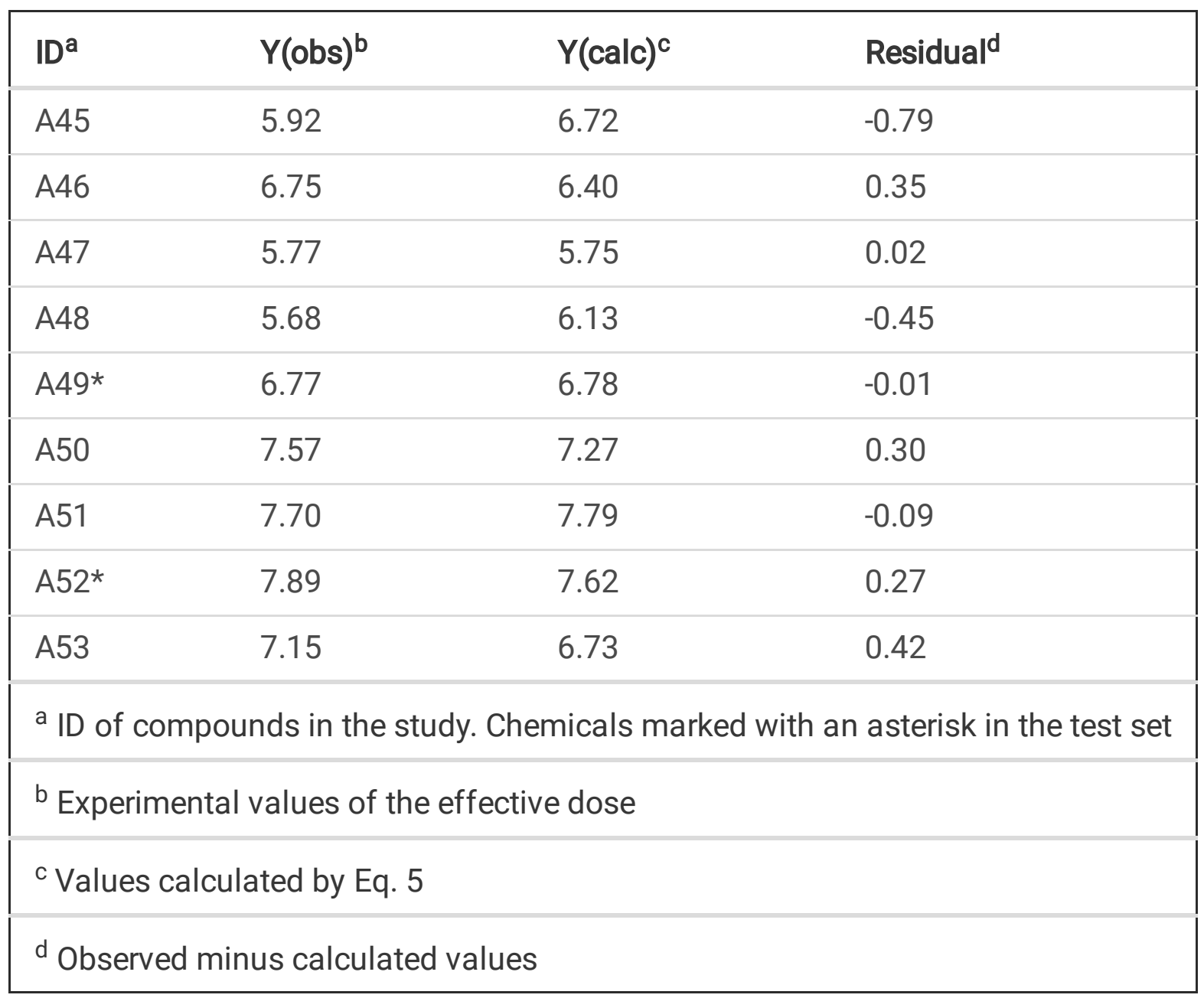

In the correlation study with the calculated descriptors, a low correlation was observed between the variables, indicating the reliable information content on each term in the equation (Table 3). The selected variables by the genetic algorithm were $\mathrm{P}$ (polarizability) $\mathrm{EH}_{2} \mathrm{O}$ (hydration energy), and q6 (partial charge of nitrogen in the common substructure 4-formamidobenzamide (Fig. 1)) (Table 4). For each one of the variables the coefficients were significant (Table 5), indicating their relative contribution to the combined prediction of the biological activity as the dependent variable. Calculating the value of the coefficients on the regression analysis we ensure a good prediction starting from the group of the independent variables (q6, $\mathrm{EH}_{2} \mathrm{O}$ and $\mathrm{P}$ ), facilitating the interpretation of the independent influence of each variable on the final equation.

Table 3: Correlation Matrix of model variables

\begin{tabular}{llll} 
& q6 $^{\mathrm{a}}$ & $\mathrm{EH}^{2} \mathbf{b}^{\mathrm{b}}$ & $\mathbf{P}^{\mathrm{c}}$ \\
\hline q6 & 1 & 0.44 & 0.13 \\
\hline EH20 & 0.44 & 1 & 0.05 \\
\hline P & 0.13 & 0.05 & 1
\end{tabular}

a Partial charge on atom N7 of the common substructure of the studied compound 
b Hydration energy

c Polarizability

Page 15/42 
Table 4

Quantum and physicochemical parameters values included in the QSAR model of 53 $\mathrm{V} 2 \mathrm{R}$ antagonist in the dataset

\begin{tabular}{|c|c|c|c|}
\hline ID & $q 6^{a}$ & $\mathrm{EH} 2 \mathrm{O}^{\mathrm{b}}$ & $\mathrm{P}^{\mathrm{c}}$ \\
\hline $\mathrm{A} 01$ & -0.73 & -5.80 & 49.72 \\
\hline A02 & -0.72 & -10.78 & 57.51 \\
\hline A03 & -0.73 & -5.38 & 36.87 \\
\hline A04 & -0.72 & -7.59 & 42.96 \\
\hline A05 & -0.74 & -14.07 & 42.74 \\
\hline A06 & -0.75 & -10.51 & 38.19 \\
\hline $\mathrm{A} 07$ & -0.73 & -5.30 & 36.87 \\
\hline A08 & -0.72 & -7.72 & 42.86 \\
\hline A09 & -0.74 & -13.88 & 42.74 \\
\hline A10 & -0.75 & -9.74 & 43.50 \\
\hline A11 & -0.73 & -8.64 & 49.81 \\
\hline A12 & -0.52 & -3.44 & 51.56 \\
\hline A13 & -0.74 & -9.63 & 43.50 \\
\hline A14 & -0.74 & -10.11 & 43.50 \\
\hline A15 & -0.74 & -7.74 & 42.96 \\
\hline A16 & -0.74 & -10.63 & 49.24 \\
\hline A17 & -0.74 & -5.86 & 49.72 \\
\hline A18 & -0.73 & -9.32 & 41.67 \\
\hline A19 & -0.73 & -7.15 & 49.72 \\
\hline A20 & -0.72 & -7.00 & 50.36 \\
\hline A21 & -0.73 & -11.05 & 51.16 \\
\hline A22 & -0.74 & -6.94 & 42.86 \\
\hline
\end{tabular}

${ }^{\text {a }}$ Partial charge on atom N7 of the common substructure of the studied compounds

b Hydration energy

c Polarizability 


\begin{tabular}{|c|c|c|c|}
\hline ID & $q 6^{a}$ & $\mathrm{EH} 2 \mathrm{O}^{\mathrm{b}}$ & $P^{c}$ \\
\hline A23 & -0.73 & -8.29 & 43.50 \\
\hline A24 & -0.71 & -12.19 & 42.74 \\
\hline A25 & -0.72 & -7.25 & 44.89 \\
\hline A26 & -0.74 & -8.15 & 41.03 \\
\hline A27 & -0.73 & -11.01 & 51.57 \\
\hline A28 & -0.73 & -6.94 & 42.86 \\
\hline A29 & -0.73 & -5.77 & 44.70 \\
\hline A30 & -0.74 & -7.79 & 42.96 \\
\hline A31 & -0.74 & -6.94 & 42.86 \\
\hline A32 & -0.73 & -6.15 & 46.53 \\
\hline A33 & -0.74 & -5.82 & 49.72 \\
\hline A34 & -0.74 & -9.86 & 40.32 \\
\hline A35 & -0.74 & -7.08 & 47.89 \\
\hline A36 & -0.77 & -7.44 & 47.89 \\
\hline A37 & -0.72 & -7.40 & 44.89 \\
\hline A38 & -0.84 & -13.07 & 52.99 \\
\hline A39 & -0.73 & -8.18 & 55.95 \\
\hline A40 & -0.73 & -7.10 & 44.22 \\
\hline A41 & -0.73 & -8.29 & 43.50 \\
\hline A42 & -0.73 & -6.61 & 51.56 \\
\hline A43 & -0.73 & -5.05 & 53.39 \\
\hline A44 & -0.73 & -10.05 & 50.03 \\
\hline A45 & -0.74 & -5.95 & 44.70 \\
\hline A46 & -0.77 & -10.96 & 44.22 \\
\hline
\end{tabular}

a Partial charge on atom N7 of the common substructure of the studied compounds

b Hydration energy

${ }^{c}$ Polarizability 


\begin{tabular}{|llll|}
\hline ID & q6 $^{\text {a }}$ & EH20 & Pc \\
\hline A47 & -0.74 & -9.13 & 40.55 \\
\hline A48 & -0.72 & -8.19 & 43.50 \\
\hline A49 & -0.73 & -6.21 & 46.05 \\
\hline A50 & -0.72 & -6.53 & 49.81 \\
\hline A51 & -0.74 & -6.31 & 51.74 \\
\hline A52 & -0.72 & -6.18 & 51.74 \\
\hline A53 & -0.71 & -11.09 & 49.60 \\
\hline a Partial charge on atom N7 of the common substructure of the studied compounds \\
\hline b Hydration energy & & \\
\hline c Polarizability & & \\
\hline
\end{tabular}

Table 5: Coefficient Analysis

\begin{tabular}{|llllll|}
\hline Predictor & Coef. $^{a}$ & Stdev $^{b}$ & $95 \%$ Conf. $^{c}$ & t-ratio $^{d}$ & $\mathbf{p}^{\mathrm{e}}$ \\
\hline Constant & -5.84 & 1.42 & 2.89 & -4.12 & 0.0002 \\
\hline q6 & -7.97 & 1.76 & 3.58 & -4.54 & 0.0001 \\
\hline EH2O & 0.10 & 0.03 & 0.06 & 3.28 & 0.0022 \\
\hline P & 0.16 & 0.01 & 0.03 & 12.15 & 0.0000 \\
\hline
\end{tabular}

${ }^{a}$ Constant and coefficients of the model variables

${ }^{b}$ Standard deviation

${ }^{\mathrm{c}}$ Confidence Interval

d Estimate divided by standard error

${ }^{\mathrm{e}}$ Level of significance

The variable q6 represents the partial charge of the N7 in the 4-formamidobenzamide common substructure, involved on an amide bond associated with the variable zone of the compounds. The partial charge $q 6$ is the most negative, with the highest module value for all the studied charges, having a fully negative value range and a negative coefficient on Eq. 5, could indicate the favorable tendency of an increased antagonist activity with more negative values of q6. It could be explained by the fact that N7 is involved in a hydrogen bond, or because it just reflects the variation of partial charge depending on the 
nature of substituent R1. The partial charge q6, calculated only for the common substructure is -0.712 and the substituent on $\mathrm{R} 1$ is making it more negative, except for compounds $\mathrm{A} 53, \mathrm{~A} 24$, and $\mathrm{A} 12$. In the case of $A 12$, the N7 is substituted by a methyl, which has a strong inductive effect (+i) over the nitrogen. Compounds A53 and A24 both have a benzene ring with a nitro substituting in the ortho position as R1. The common substructure in most compounds is formed entirely by a conjugated system with a substituted benzene ring as R1, which might contribute to a whole conjugated system. If we compare the compounds by the position of the substituent on the benzene ring at R1, we observe a lower partial charge on q6 associated with an ortho substituent, calling for a combination of steric and electronic factors, where the ortho substituent could break the conjugation planarity.

Hydration energy $\left(\mathrm{EH}_{2} \mathrm{O}\right)$ is the amount of energy released when one mole of a compound is hydrated, and represents the measure of the water molecules affinity for the compound. More negative hydration energy values could be associated with more polar groups in the compound, and less negative hydration energy could be attributed to the presence of a higher number of nonpolar groups [56]. The range of values for this variable in the data set is negative and it has a positive coefficient on Eq. 5 , thus indicating that more negative values of hydration energy are unfavorable for the antagonist activity, suggesting a binding site with possible hydrophobic interactions, as more hydrophilic compounds are shown unfavorable for the activity.

Polarizability refers to the tendency of any compound to acquire an electric dipole moment in proportion to an applied electric field, on our model the Polarizability component is having a correlation coefficient of 0.7 with the $\mathrm{pIC}_{50}$, making it the descriptor with the highest correlation to the activity, being the other variables the fine adjustments necessary to improve the model in general. The range of polarizability values in the data set is positive and has a positive coefficient in Eq. 5, so it has a favorable contribution to antagonist activity.

As mentioned above, the compounds in the studied dataset have conjugated systems in their structure, and systems with delocalized $\pi$ electrons exhibit high polarizabilities. The aromatic systems' planarity with their high polarizability and multipole moment, are all factors of key importance for the 3D architecture of aromatic complexes [57]. Soft interactions like dispersion, are predominant in stacking and can be estimated from the polarizability [58]. Another possible interaction for the polarizable $\pi-$ electron cloud of aromatic rings is with cations, and polarizability is also relevant for this $\pi$-cation interaction $[59,60]$. In general, the studied compounds have three aromatic rings in their structure that could be involved in $\pi$-interactions with the residues in the receptor-binding site.

In general, the obtained QSAR model provides indications that the binding mode of V2R antagonists might fundamentally be involving hydrophobic and electron density interactions.

\subsection{The applicability domain (AD) of the QSAR model}

A QSAR model needs to show not only a good accuracy, but also some reliability for predictions of new compounds, in general these models cannot be universal and should be constrained to a defined 
chemical space, commonly known as the applicability domain (AD). The AD can be described as the physicochemical, structural or biological spatial information based on which the model training set is developed. The QSAR model applies to make predictions for new compounds within the specific domain [57]; in summary, the AD is the degree to which a QSAR model tolerates (reliably) new compounds.

A crucial problem in chemometrics and QSAR studies is the definition of the AD with a regression model. We will define it here as a squared area within \pm 2 bands for standardized residuals and a leverage threshold of $\mathrm{h}=0.23$ for inhibitory activity (Eq. 5). Thus, compounds with standardized residuals greater than 2 standard deviations will be considered unreliable. For the graphical visualization of outliers for the response (standardized residuals $>2$ ) or for the structure (leverage $>0.23$ ) in the regression model, the Williams plot for Eq. 5 is shown in Fig. 4. Of the 53 compounds in the dataset, only two compounds (A02 and A12) have a leverage higher than the critical value.

A02 (conivaptan) has the highest value of polarizability (57.51) of the dataset, while the other compounds are between 36.87 to 55.95 . A02 shows a diphenyl moiety as substituent of the amide in the common substructure, while the other compounds exhibit only a single aromatic ring or an aliphatic substituent, A02 also have a condensed 3 ring system of 3,4,5,6-tetrahydroimidazol[4,5-d][1]benzazepine, while the other compounds have only a 2 ring system. The presence of extra rings on A02, might account for the increase on the polarizability for this compound with a different electronic structure than the rest.

A12 shows the highest value of q6 (-0.52) of the dataset, while the other compounds are between of -0.71 to -0.84 , and it also has the highest value of hydration energy $\left(\mathrm{EH}_{2} \mathrm{O}\right)$, with $-3.44 \mathrm{kcal} / \mathrm{mol}$, while the other compounds are between -5.05 to $-14.07 \mathrm{kcal} / \mathrm{mol}$. Compound $\mathrm{A} 12$ show a minor difference in the common substructure with all the antagonists of this family having a methyl group as substituent for the $\mathrm{N}$ amide, directly altering the partial charge (q6) of N7 on the 4-formamidobenzamide and increasing the hydration energy value. The structure of A12 and A01 differ only in the aforementioned methyl group, A12 has a difference of 0.20 on q6, and of $2.36 \mathrm{kcal} / \mathrm{mol}$ in the value of hydration energy compared to $\mathrm{A} 01$, evidencing the influence that this single methyl group can have.

\subsection{V2R modeling}

We selected the human OX2 orexin receptor (PDB ID 4SOV) as the template for V2R modeling, as suggested by the GPCRdb template tool. The selected template has $27 \%$ of identity and $46 \%$ of similarity with $\mathrm{V} 2 \mathrm{R}$. In the corresponding alignment, the fragments corresponding to transmembrane helices and the conserved motifs are preserved (Fig. 5). In both the OX2 receptor and V2R, the natural ligand is a peptide, and the selected structure has an antagonist bound being on an inactive conformation, suitable to study the binding modes of antagonists to the V2 receptor.

To relax the obtained model in a more natural environment, it was minimized and equilibrated for 10ns in a POPC membrane, solvated and with $\mathrm{NaCl}$ added at physiological concentration $(0.15 \mathrm{M})$. At the end of the simulation the membrane parameters, like thicknesses and per lipid area, were calculated to check the correct packing. The membrane thickness (distances between phosphates of each monolayer) was 38.91 
$\AA$, and the per lipid area was $64.54 \AA^{2}$. These parameters are reasonable for a POPC membrane at $310 \mathrm{~K}$, according to experimental parameters obtained at different temperatures [62].

During the current work, another X-ray structure from the class A of GPCR was released in the Protein Data Bank (PDB ID 6TPK): the oxytocin receptor (OXTR) which is also a member of the vasopressin receptor family. Although OXTR exhibits slightly better sequence identity and similarity: $41 \%$ and $56 \%$ respectively, its lower resolution (3.20 ̊ versus $2.50 \AA$ ), a missing region (loop and helix 8), and a shorter loop (ICL3) make it a less relevant template. However, in an effort for further validation, the V2R model was compared to OXTR; the superimposition between the V2R model and OXTR is shown in Fig. 6, where we considered the two conformations of $\mathrm{V} 2 \mathrm{R}$ before and after membrane relaxation.

The RMSD values, using OXTR as reference and comparing the model before and after the membrane relaxation were $1.02 \AA$ and $1.15 \AA$ respectively. The main differences between OXTR and the models were that OXTR lacks the ICL3, a long intracellular loop involved in the interaction with the G-protein usually missing in GPCR solved structures and the helix 8, parallel to the membrane and useful for orienting the receptor in the membrane. Comparing the bundled helices, the main difference relative to the binding site is that the TM2 of relaxed model in the membrane is in the same position as that of the OXTR, while the model before relaxing has the TM2 slightly tilted towards the interior of the cavity decreasing its volume. The difference in the orientation of TM2 in the model before relaxation could be caused by the difference in the proline position in this transmembrane section between V2R and the template (Fig. 5). Proline in the middle of alpha helices cause a kink, by being unable to complete the $\mathrm{H}$-bonding chain of the helix and because of steric and/or rotameric effects keeping it out from the preferred helical geometry [63]. Proline one position earlier on the sequence of the template TM2 with respect to that of the model, can affect the orientation of the kink and result on a different orientation in the model. This odd orientation could later be corrected during the relaxation in the membrane showing this process as very favorable.

The comparison between the V2R model after relaxation and OXTR brings more confidence in the model's quality and the protocol used for relaxation.

\subsection{V2R-antagonist complexes}

Visual inspection of the binding site revealed that the side chains of residues W99 and F307 are occluding the entrance of the binding site, therefore these two residues were considered as flexible for the molecular docking. To improve docking results other residues of the binding site (Q96, F105 and K116) were also considered as flexible.

The antagonists selected as ligands for the molecular docking were: mozavaptan (A01), conivaptan (A02), and tolvaptan. Tolvaptan also shares the common substructure of the studied compound series for the QSAR model and it is the only drug approved to treat Polycystic Kidney Disease. All the rotatable bonds of the ligands were flexible.

The 50 complexes for each antagonist obtained by molecular docking were clustered for analysis. Three orientations of the antagonists in the binding site were identified and shown in Fig. 7. The first with the 
condensed ring of the antagonist toward TM2 and TM7 (CR-27), the second with the condensed ring towards TM5 and TM6 (CR-56) and the last one with the condensed ring towards the entrance of the cavity (CR-UP). The binding energies of the complexes obtained for the three identified conformations differ by less than $1 \mathrm{kcal} / \mathrm{mol}$, in each of the antagonists studied. This difference in the energy value is lower than the standard error of the Autodock Vina scoring function [45], so we might need more studies to select the best conformation.

We expect a good antagonist to bind with high affinity to the receptor binding site but failing to activate it, blocking the access of any agonist to the binding site. A study with meta- dynamics enhanced sampling revealed the existence of three binding sub-sites for V2R, proposed to respond to the vasopressin entry pathway [64]. The compounds that bound in both the vestibule and the intermediate sites block the access to the orthosteric site so that, an agonist will never be able to bind, if there is an antagonist already bound to any of the non-activating sites. Two of the antagonists studied by Saleh et al [64], with high structural similarity to those in this study, were predicted to bind to vestibule site for V2R and intermediate site for $\mathrm{V} 1 \mathrm{aR}$, so it is to be expected that the antagonists in our study are located in one of these sites. Therefore, we eliminated from our subsequent analysis the CR-UP conformation, showing the antagonist penetrating deeper into the cavity with part of it located in the orthosteric site.

To study the stability of the compounds in the binding site and make a better estimation of the binding energy, a molecular dynamic simulation of the best complex of the two remaining orientation was performed. Figure 8 show the RMSD for the two different conformations of the three studied antagonists. The conformations of the studied antagonists tend to stabilize along the molecular dynamics simulations, being CR-27 the conformation with lower RMSD for each of the antagonist. For mozavaptan, the conformation CR-56 is the conformation with more fluctuations along the trajectory and it has the highest RMSD value among all antagonist conformations. The change in the antagonist's conformation for the six complexes on the molecular dynamics simulation is show in Fig. 9. The two-representative conformation for each antagonist are represented from left to right (CR-27 and CR-56 respectively). Tolvaptan is represented in green, conivaptan in blue and mozavaptan in pink. The starting conformation at $0 \mathrm{~ns}$ is represented by the light colored ligand and the final conformation at $50 \mathrm{~ns}$ by the dark colored ligand. Mozavaptan CR-56 conformation showed a significant change in the orientation of the antagonist with respect to the starting conformation, also reflected in the high RMSD value observed for this conformation. For this reason, this conformation was eliminated from the subsequent analysis.

In order to predict which binding conformation could be the best for each antagonist, we estimated the binding free energy variation using LIE-D method. This method is flexible enough to consider different interaction patterns even though the ligands share some common chemical scaffolds and are bound to the same protein receptor [53].

For tolvaptan, the conformation with the best binding free energy is tolvaptan CR-27 $(-14.34 \mathrm{kcal} / \mathrm{mol})$ with over $2 \mathrm{kcal} / \mathrm{mol}$ of difference with $\mathrm{CR}-56$, that was thus discarded from further analysis. The conformations of conivaptan have similar energies between them, so it is not possible to select which of 
the two conformations is the best using this criterion. The inhibition constant $\left(\mathrm{K}_{i}\right)$ was calculated from the estimated binding free energy for these conformations and compared with experimental values (Table 6). The values of the calculated $\mathrm{K}_{i}$ follow the same trend than the experimental inhibition constants $[65,66]$, except for the tolvaptan, with the $K_{i}$ for conformation CR-27 one order lower than the experimental value and the $\mathrm{K}_{i}$ conformation $\mathrm{CR}-56$ one order higher, suggesting as expected that CR-27 was the best conformation for tolvaptan.

Table 6: Antagonist-V2R estimated binding free energy $(\boldsymbol{\Delta} \mathbf{G}(\mathbf{k c a l} / \mathrm{mol}))$ by LIE-D method and estimated inhibition constant $\left(\mathrm{K}_{\mathrm{i}}\right)$

\begin{tabular}{llllll} 
Antagonist & \multicolumn{2}{l}{$\Delta \mathrm{G}(\mathrm{kcal} / \mathrm{mol})$} & $\mathrm{K}_{i}(\mathrm{nM})$ & & \\
\cline { 2 - 6 } & CR_27 & CR_56 & CR_27 & CR_56 & experimental \\
\hline conivaptan & -12.94 & -12.98 & 0.70 & 0.66 & $0.36[65]$ \\
\hline mozavaptan & -11.60 & - & 6.17 & - & $9.42[66]$ \\
\hline tolvaptan & -14.23 & -11.56 & 0.09 & 6.65 & $0.43[66]$
\end{tabular}

The binding free energy and $\mathrm{K}_{i}$ values obtained for the different conformations of the antagonists bound to V2R allowed us to select the CR-27 conformations (or the CR-56 conformation for conivaptan only) as the possible binding modes of these antagonists to V2R. From these conformations, the analysis of the main antagonist-receptor complex interactions can be carried out.

\subsection{Interactions analysis of the best antagonist-V2R complexes}

The antagonists must block the access or interact with those residues favoring the union of any agonist for the receptor activation, and/or also sterically blocking the residues involved in triggering the activation mechanism. The most relevant contacts between the antagonist and the receptor are summarized in Table 7. The interactions observed for the mozavaptan and tolvaptan complexes are very similar, while conivaptan interacts with a greater number of residues, since it is a compound with a greater volume than those mentioned above.

Table 7: Most relevant contacts for the Antagonist-Receptor complexes. The numbers represent the percent of frames with the contact present in the trajectory. Only those contacts that were observed in more than $50 \%$ of the trajectory are represented with numbers. 
MVP-CR -27 CVP-CR-27 CVP-CR-56 TVP-CR-27

\begin{tabular}{lllll}
\hline Q92 & - & 97.6 & - & - \\
\hline Q96 & 95.0 & 91.3 & 97.4 & 99.9 \\
\hline W99 & 99.1 & 76.8 & - & 97.8 \\
\hline F105 & 95.0 & 54.4 & 70.6 & 98.0 \\
\hline K116 & 98.1 & 64.9 & 96.9 & 99.5 \\
\hline Q119 & - & 59.2 & 96.8 & - \\
\hline F178 & 87.2 & - & - & 92.2 \\
\hline C192 & 99.3 & - & - & 100 \\
\hline A194 & 93.2 & 95.9 & 59.1 & 94.6 \\
\hline Y205 & - & 93.1 & - & - \\
\hline V206 & - & 93.6 & 86.1 & - \\
\hline Q291 & - & 63.3 & 99.0 & - \\
\hline F307 & 97.9 & 90.9 & 98.3 & 96.5 \\
\hline L310 & - & 68.0 & 82.1 & - \\
\hline M311 & - & 61.4 & 87.1 & 87.5
\end{tabular}

In the analysis of the obtained complexes, there are some common interactions involving the hydrophobic (C192, A194, L310, M311), aromatic (W99, F105, F178, F307) and polar (Q96, K116, Q119, Q291) residues. The residues Q96, Q119, Q291, K116 are highly conserved in all the AVP and OXT receptors' family, and are known to have a key role in agonist binding [67]. Previous studies with V1aR suggest that the residues Q96, Q119, Q291 are K116 are specifically involved with the ligand binding process but do not intrinsically modulate the efficacy of the functional response [67]. An analysis of the presence of $\mathrm{H}$-bonds along the molecular dynamic simulation was performed to study the nature of the interactions between the antagonists and these polar residues of the receptor. The percentage of frames of the trajectory with a determined number of $\mathrm{H}$-bonds is shown in the Table 8 . In all the studied conformations the occurrence of $\mathrm{H}$-bonds is low, approximately between 13 and $38 \%$, although interactions with some polar residues are observed in greater percentages of the trajectory (Table 7), which suggests other kind of interactions. In the case of K116, the positively charged $\varepsilon$-amino group can also interact with aromatic rings. This $\pi$-cation interaction was detected between K116 and a ring of the studied antagonists (Fig. 10 and Supplementary Information Figures S1-S3). 
Table 8: Analysis of H-bond between antagonist-V2R H-bonds of the studied conformations. The numbers represent the occurrence percentage of $\mathrm{H}$-bonds along the molecular dynamic simulations

\begin{tabular}{|c|c|c|c|c|c|}
\hline \multirow[t]{2}{*}{ No. H-bonds } & \multicolumn{5}{|c|}{ Conformations } \\
\hline & MVP-CR27 & CVP-CR27 & CVP-CR56 & TVP-CR27 & TVP-CR56 \\
\hline 0 & 76.12 & 63.68 & 61.72 & 85.50 & 59.86 \\
\hline 1 & 22.46 & 31.28 & 35.60 & 13.64 & 33.30 \\
\hline 2 & 1.34 & 4.74 & 2.66 & 0.84 & 6.40 \\
\hline 3 & 0.08 & 0.30 & 0.02 & 0.02 & 0.44 \\
\hline
\end{tabular}

The antagonists interact with the aromatic residues W99, F105, F178 and F307. These residues are not directly involved in the receptor activation, and they are near or at the entrance of the binding site, suggesting that the antagonist binding site might not be located deep into the cavity. The fact that the antagonists interact with residues near the entrance of the cavity agrees with the binding sites predicted by Saleh et al [64].

Some of these residues have been shown to be involved in vasopressin binding. W99 plays a fundamental role in stabilizing the vasopressin/receptor interactions responsible for the high-affinity binding of agonists to the V2 receptor and receptor selectivity. A mutation of W99 (W99R) greatly impaired the binding properties of the receptor and had a minor effect on its intracellular routing [68]. Other important residue for AVP binding is F105, the mutation F105V was reported to show cell surface expression and a maximal AVP-induced cAMP formation (Vmax) comparable to the wild type, but with a reduced ligand binding ability $[69,70]$.

An interesting interaction for the V2R antagonists is with $\mathrm{F} 307$, a non-conserved residue in vasopressin/oxytoxin family since $\mathrm{V} 1 \mathrm{aR}$ has a threonine in this position. The relevance of this interaction is because some antagonists could bind to both V2R and V1aR due to the similarity of its binding site, but the interaction with F307 would be unique for V2R making it attractive for the design of antagonists having less selectivity for $\mathrm{V} 1 \mathrm{aR}$.

Other antagonist-receptor interactions found were with residues C192, A194 and M311. While C192 and A194 are conserved among the entire family, M311 is not, seeming to cooperate in the selective binding of some antagonists [71]. The M311V mutation in the TM7 of V2R has impaired the ligand capacity and binding [72] suggesting that in V2R the residue M311 could take part in the binding of peptide agonists $[70,73]$

Taking into account the interactions and the estimated binding free energy, we considered CR-27 conformation (and CR-56 for conivaptan) as the best for the three antagonists studied by molecular dynamics. Figure 10 shows the CR-27 conformation of tolvaptan in complex with V2R. 
In general, the main interactions observed here are involved in the binding of ligands to V2R, but are not involved on the receptor activation, which suggest that the studied conformations of the antagonists can block the binding of agonists and unable to activate the receptor. The presence of few $\mathrm{H}$-bonds with polar residues and the other interactions observed are with aromatic residues and non-polar residues suggest that the main antagonist-receptor interactions are mostly hydrophobic in nature and could involve $\pi-$ clouds.

\section{Conclusion}

In summary, two computational approaches, ligand and receptor based were developed to study the physicochemical properties relevant to the biological activity of $\mathrm{V} 2 \mathrm{R}$ antagonists and to predict their binding mode to V2R. The proposed QSAR model allows us to clarify the contribution of three molecular descriptors to the biological activity. Our model described the antagonist activity in correlation with polarizability, hydration energy and partial charge on atom N7, explaining the molecular properties contributing to the antagonist-receptor interaction and relevant to the antagonist activity, which is also in agreement with the binding modes for the complexes obtained by molecular docking and molecular dynamics simulation.

A good quality model based on the structure of OX2 orexin receptor was obtained and used to estimate the antagonist orientations in the binding site of $\mathrm{V} 2 \mathrm{R}$. The conformations of studied antagonist were analyzed by molecular dynamics. In general, the CR-27 conformation is considered as the best conformation for the antagonist binding (through interaction analysis and binding free energy estimation). Most of the relevant interactions observed along the molecular dynamics simulation involve the electronic density by the interaction of the antagonist rings mainly with the aromatic residues (W99, F105, F178 and F307) and the positively charged residue K116, which is in correspondence with what is expected according to the polarizability variable of our QSAR model. Other relevant interactions are hydrophobic in nature (A194 and M311) which agree with the expected effect of the hydration energy to the antagonist activity in the QSAR model.

The results obtained by both developed approaches are in fair agreement and contribute to a better understanding of $\mathrm{V} 2 \mathrm{R}$ antagonism. These results represent a step forward for the efficient search of potential new V2R antagonist molecules.

\section{Abbreviations}

GPCR: G Protein Coupled Receptor; PKD: Polycystic Kidney Disease ;ADPKD: Autosomal Dominant Polycystic Kidney Disease; AVP: arginine vasopressin; V2R vasopressin V2 receptor; SIADH: Syndrome of inappropriate antidiuretic hormone secretion; ADH: antidiuretic hormone; QSAR: Quantitative Structure Activity Relationship; GA: Genetic Algorithm; MLR: Multiple Linear Regression; POPC: 1-palmitoyl-2-oleoylsn-glycero-3-phosphocholine; RMSD: Root mean-square deviation; NPT: constant number of particles, pressure, and temperature; PDB: Protein Data Bank. 


\section{Declarations}

\section{Funding}

The authors received no specific funding for this work.

\section{Conflicts of interest}

The authors confirm the article content has no conflict of interest.

\section{Availability of data and material}

All the information about datasets during and/or analyzed during the current research are included in the manuscript, additional file and other required data is available from the corresponding author on reasonable request.

\section{Code availability}

N/A

\section{Authors' contributions}

A.N.V. contributed with the descriptor calculation, QSAR model, receptor modeling, docking, MD simulations, analysis, data interpretation, generating figures and manuscript writing. Y.M.A.G contributed with the QSAR methodology, applicability domain, analysis, data interpretation and manuscript writing. R.E.R.F. contributed with the receptor based methodology, analysis, data interpretation and manuscript writing. F.L. contributed with the MD simulations methodology, data interpretation, generating figures and manuscript writing. L.A.M.C contributed with project development, data interpretation and manuscript edition.

\section{References}

1. Yamaguchi T, Wallace DP, Magenheimer BS et al (2004) Calcium restriction allows cAMP activation of the B-Raf/ERK pathway, switching cells to a cAMP-dependent growth-stimulated phenotype. J Biol Chem 279:40419-40430. https://doi.org/10.1074/jbc.M405079200

2. Torres VE, Wang X, Qian Q et al (2004) Effective treatment of an orthologous model of autosomal dominant polycystic kidney disease. Nat Med 10:363-364. https://doi.org/10.1038/nm1004

3. Grantham JJ (2014) Rationale for early treatment of polycystic kidney disease. Pediatr Nephrol Berl Ger. https://doi.org/10.1007/s00467-014-2882-8

4. Decaux G, Soupart A, Vassart G (2008) Non-peptide arginine-vasopressin antagonists: the vaptans. The Lancet 371:1624-1632. https://doi.org/10.1016/S0140-6736(08)60695-9

5. Bankir L, Bichet DG, Morgenthaler NG (2017) Vasopressin: physiology, assessment and osmosensation. J Intern Med 282:284-297. https://doi.org/10.1111/joim.12645 
6. Thibonnier M, Coles P, Thibonnier A, Shoham M (2002) Molecular pharmacology and modeling of vasopressin receptors. Prog Brain Res 139:179-196. https://doi.org/10.1016/s0079-6123(02)390162

7. Facciorusso A, Amoruso A, Neve $\mathrm{V}$ et al (2014) Role of vaptans in the management of hydroelectrolytic imbalance in liver cirrhosis. World J Hepatol 6:793-799. https://doi.org/10.4254/wjh.v6.i11.793

8. Aihara M, Fujiki H, Mizuguchi $\mathrm{H}$ et al (2014) Tolvaptan delays the onset of end-stage renal disease in a polycystic kidney disease model by suppressing the increases in kidney volume and renal injury. $J$ Pharmacol Exp Ther. https://doi.org/10.1124/jpet.114.213256

9. Boertien WE, Meijer E, de Jong PE et al (2015) Short-term Effects of Tolvaptan in Individuals With Autosomal Dominant Polycystic Kidney Disease at Various Levels of Kidney Function. Am J Kidney Dis Off J Natl Kidney Found. https://doi.org/10.1053/j.ajkd.2014.11.010

10. Kelsey R (2013) Polycystic kidney disease: Tolvaptan in ADPKD-TEMPO 3:4 trial results. Nat Rev Nephrol 9:1. https://doi.org/10.1038/nrneph.2012.236

11. Manning M, Stoev S, Chini B et al (2008) Peptide and non-peptide agonists and antagonists for the vasopressin and oxytocin V1a, V1b, V2 and OT receptors: research tools and potential therapeutic agents. Prog Brain Res 170:473-512. https://doi.org/10.1016/S0079-6123(08)00437-8

12. Manning M, Misicka A, Olma A et al (2012) Oxytocin and Vasopressin Agonists and Antagonists as Research Tools and Potential Therapeutics. J Neuroendocrinol 24:609-628. https://doi.org/10.1111/j.1365-2826.2012.02303.x

13. Rondon-Berrios H, Berl T (2016) Vasopressin receptor antagonists: Characteristics and clinical role. Best Pract Res Clin Endocrinol Metab 30:289-303. https://doi.org/10.1016/j.beem.2016.02.004

14. Yamamura $\mathrm{Y}$, Ogawa $\mathrm{H}$, Yamashita $\mathrm{H}$ et al (1992) Characterization of a novel aquaretic agent, OPC31260 , as an orally effective, nonpeptide vasopressin V2 receptor antagonist. $\mathrm{Br} \mathrm{J}$ Pharmacol 105:787-791. https://doi.org/10.1111/j.1476-5381.1992.tb09058.x

15. Ohnishi A, Orita Y, Okahara R et al (1993) Potent aquaretic agent. A novel nonpeptide selective vasopressin 2 antagonist (OPC-31260) in men. J Clin Invest 92:2653-2659. https://doi.org/10.1172/JCl116881

16. Drugs@FDA: FDA-Approved Drugs. https://www.accessdata.fda.gov/scripts/cder/daf/index.cfm. Accessed 23 Sep 2020

17. Ranieri M, Di Mise A, Tamma G, Valenti G (2019) Vasopressin-aquaporin-2 pathway: recent advances in understanding water balance disorders. F1000Research 8:. https://doi.org/10.12688/f1000research.16654.1

18. El Boustany R (2018) Vasopressin and Diabetic Kidney Disease. Ann Nutr Metab 72:17-20. https://doi.org/10.1159/000488124

19. Izumi Y, Miura K, Iwao H (2014) Therapeutic potential of vasopressin-receptor antagonists in heart failure. J Pharmacol Sci 124:1-6. https://doi.org/10.1254/jphs.13r13cp 
20. Gassanov N, Semmo N, Semmo M et al (2011) Arginine vasopressin (AVP) and treatment with arginine vasopressin receptor antagonists (vaptans) in congestive heart failure, liver cirrhosis and syndrome of inappropriate antidiuretic hormone secretion (SIADH). Eur J Clin Pharmacol 67:333346. https://doi.org/10.1007/s00228-011-1006-7

21. Bodor N, Gabanyi Z, Wong CK (1989) A new method for the estimation of partition coefficient. J Am Chem Soc 111:3783-3786. https://doi.org/10.1021/ja00193a003

22. Hasel W, Hendrickson TF, Still WC (1988) A rapid approximation to the solvent accessible surface areas of atoms. Tetrahedron Comput Methodol 1:103-116. https://doi.org/10.1016/08985529(88) $90015-2$

23. Miller KJ (1990) Additivity methods in molecular polarizability. J Am Chem Soc 112:8533-8542. https://doi.org/10.1021/ja00179a044

24. Parthasarathi R, Subramanian V, Roy DR, Chattaraj PK (2004) Electrophilicity index as a possible descriptor of biological activity. Bioorg Med Chem 12:5533-5543. https://doi.org/10.1016/j.bmc.2004.08.013

25. Frisch M, Truck G, Schlegel $\mathrm{H}$ et al (2009) Gaussian 09, Revision A.1. Gaussian Inc Wallingford CT 26. Froimowitz M (1993) HyperChem: a software package for computational chemistry and molecular modeling. BioTechniques

27. McFarland J, Gans D (1995) Multivariate Data Analysis of Chemical and Biological Data. Cluster Significance Analysis. In: Chemometrics Methods in Molecular Design. VCH Publishers, Inc, New York, pp 295-308

28. Alvarez-Ginarte YM, Montero-Cabrera LA, García-de la Vega JM et al (2013) Integration of ligand and structure-based virtual screening for identification of leading anabolic steroids. J Steroid Biochem Mol Biol 138:348-358. https://doi.org/10.1016/j.jsbmb.2013.07.004

29. Yu S, Tranchevent L, Liu X et al (2012) Optimized Data Fusion for Kernel k-Means Clustering. IEEE Trans Pattern Anal Mach Intell 34:1031-1039. https://doi.org/10.1109/TPAMI.2011.255

30. Weiß CH (2007) StatSoft, Inc., Tulsa OK: STATISTICA, Version 8. AStA Adv Stat Anal 91:339-341. https://doi.org/10.1007/s10182-007-0038-x

31. Alvarez-Ginarte YM, Crespo R, Montero-Cabrera LA et al (2005) A novel in-silico approach for QSAR Studies of Anabolic and Androgenic Activities in the 17ß-hydroxy-5a-androstane Steroid Family. QSAR Comb Sci 24:218-226. https://doi.org/10.1002/qsar.200430889

32. Liu P, Long W (2009) Current mathematical methods used in QSAR/QSPR studies. Int J Mol Sci 10:1978-1998. https://doi.org/10.3390/ijms10051978

33. Pourbasheer E, Aalizadeh R, Ganjali MR, Norouzi P (2013) QSAR study of IKK $\beta$ inhibitors by the genetic algorithm: multiple linear regressions. Med Chem Res 23:57-66. https://doi.org/10.1007/s00044-013-0611-7

34. Pourbasheer E, Ahmadpour S, Zare-Dorabei R, Nekoei M (2017) Quantitative structure activity relationship study of p38a MAP kinase inhibitors. Arab J Chem 10:33-40. https://doi.org/10.1016/j.arabjc.2013.05.009 
35. Tropsha A (2010) Best Practices for QSAR Model Development, Validation, and Exploitation. Mol Inform 29:476-488. https://doi.org/10.1002/minf.201000061

36. Gramatica P (2020) Principles of QSAR Modeling: Comments and Suggestions From Personal Experience. Int J Quant Struct-Prop Relatsh IJQSPR 5:61-97. https://doi.org/10.4018/IJQSPR.20200701.oa1

37. Oliveira DB de, Gaudio AC (2001) BuildQSAR: A New Computer Program for QSAR Analysis. Quant Struct-Act Relatsh 19:599-601. https://doi.org/10.1002/1521-3838(200012)19:6<599::AIDQSAR599>3.0.CO;2-B

38. Larkin MA, Blackshields G, Brown NP et al (2007) Clustal W and Clustal X version 2.0. Bioinforma Oxf Engl 23:2947-2948. https://doi.org/10.1093/bioinformatics/btm404

39. Krieger E, Koraimann G, Vriend G (2002) Increasing the precision of comparative models with YASARA NOVA-a self-parameterizing force field. Proteins 47:393-402. https://doi.org/10.1002/prot.10104

40. Humphrey W, Dalke A, Schulten K (1996) VMD: visual molecular dynamics. J Mol Graph 14:33-38. https://doi.org/10.1016/0263-7855(96)00018-5 27-28.

41. Stansfeld PJ, Goose JE, Caffrey M et al (2015) MemProtMD: Automated Insertion of Membrane Protein Structures into Explicit Lipid Membranes. Struct Lond Engl 1993 23:1350-1361. https://doi.org/10.1016/j.str.2015.05.006

42. Phillips JC, Braun R, Wang W et al (2005) Scalable molecular dynamics with NAMD. J Comput Chem 26:1781-1802. https://doi.org/10.1002/jcc.20289

43. Guixà-González R, Rodriguez-Espigares I, Ramírez-Anguita JM et al (2014) MEMBPLUGIN: studying membrane complexity in VMD. Bioinforma Oxf Engl 30:1478-1480.

https://doi.org/10.1093/bioinformatics/btu037

44. Morris GM, Huey R, Lindstrom W et al (2009) AutoDock4 and AutoDockTools4: Automated Docking with Selective Receptor Flexibility. J Comput Chem 30:2785-2791.

https://doi.org/10.1002/jcc.21256

45. Trott O, Olson AJ (2010) AutoDock Vina: Improving the speed and accuracy of docking with a new scoring function, efficient optimization, and multithreading. J Comput Chem 31:455-461. https://doi.org/10.1002/jcc. 21334

46. Pettersen EF, Goddard TD, Huang CC et al (2004) UCSF Chimera-a visualization system for exploratory research and analysis. J Comput Chem 25:1605-1612. https://doi.org/10.1002/jcc.20084

47. Durrant JD, McCammon JA (2011) BINANA: A Novel Algorithm for Ligand-Binding Characterization. J Mol Graph Model 29:888-893. https://doi.org/10.1016/j.jmgm.2011.01.004

48. OpenEye Scientific Software. Cheminformatics Software | Molecular Modeling Software I OpenEye Scientific. https://www.eyesopen.com/. Accessed 1 Jun 2021

49. Best RB, Zhu X, Shim J et al (2012) Optimization of the additive CHARMM all-atom protein force field targeting improved sampling of the backbone $\varphi, \psi$ and side-chain $\chi 1$ and $\chi 2$ dihedral angles. $J$ Chem 
Theory Comput 8:3257-3273. https://doi.org/10.1021/ct300400x

50. Jo S, Kim T, Iyer VG, Im W (2008) CHARMM-GUI: A web-based graphical user interface for CHARMM. J Comput Chem 29:1859-1865. https://doi.org/10.1002/jcc.20945

51. Lee J, Cheng X, Swails JM et al (2016) CHARMM-GUI Input Generator for NAMD, GROMACS, AMBER, OpenMM, and CHARMM/OpenMM Simulations Using the CHARMM36 Additive Force Field. J Chem Theory Comput 12:405-413. https://doi.org/10.1021/acs.jctc.5b00935

52. Almlöf M, Carlsson J, Åqvist J (2007) Improving the Accuracy of the Linear Interaction Energy Method for Solvation Free Energies. J Chem Theory Comput 3:2162-2175. https://doi.org/10.1021/ct700106b

53. Miranda WE, Noskov SY, Valiente PA (2015) Improving the LIE Method for Binding Free Energy Calculations of Protein-Ligand Complexes. J Chem Inf Model 55:1867-1877. https://doi.org/10.1021/acs.jcim.5b00012

54. Hansson T, Marelius J, Åqvist J (1998) Ligand binding affinity prediction by linear interaction energy methods. J Comput Aided Mol Des 12:27-35. https://doi.org/10.1023/A:1007930623000

55. Yu S, Tranchevent L, Liu X et al (2012) Optimized Data Fusion for Kernel k-Means Clustering. IEEE Trans Pattern Anal Mach Intell 34:1031-1039. https://doi.org/10.1109/TPAMI.2011.255

56. Schauperl M, Podewitz M, Waldner BJ, LiedI KR (2016) Enthalpic and Entropic Contributions to Hydrophobicity. J Chem Theory Comput 12:4600-4610. https://doi.org/10.1021/acs.jctc.6b00422

57. Riley KE, Hobza P (2013) On the Importance and Origin of Aromatic Interactions in Chemistry and Biodisciplines. Acc Chem Res 46:927-936. https://doi.org/10.1021/ar300083h

58. Mignon P, Loverix S, Steyaert J, Geerlings $P(2005)$ Influence of the $\pi-\pi$ interaction on the hydrogen bonding capacity of stacked DNA/RNA bases. Nucleic Acids Res 33:1779-1789. https://doi.org/10.1093/nar/gki317

59. Cubero E, Luque FJ, Orozco M (1998) Is polarization important in cation- $\pi$ interactions? Proc Natl Acad Sci 95:5976-5980. https://doi.org/10.1073/pnas.95.11.5976

60. Marshall MS, Steele RP, Thanthiriwatte KS, Sherrill CD (2009) Potential Energy Curves for Cation $-\pi$ Interactions: Off-Axis Configurations Are Also Attractive. J Phys Chem A 113:13628-13632. https://doi.org/10.1021/jp906086x

61. Roy K, Kar S, Das RN (2015) Chap. 7 - Validation of QSAR Models. In: Roy K, Kar S, Das RN (eds) Understanding the Basics of QSAR for Applications in Pharmaceutical Sciences and Risk Assessment. Academic Press, Boston, pp 231-289

62. Kučerka N, Nieh M-P, Katsaras J (2011) Fluid phase lipid areas and bilayer thicknesses of commonly used phosphatidylcholines as a function of temperature. Biochim Biophys Acta 1808:2761-2771. https://doi.org/10.1016/j.bbamem.2011.07.022

63. von Heijne G (1991) Proline kinks in transmembrane a-helices. J Mol Biol 218:499-503. https://doi.org/10.1016/0022-2836(91)90695-3 
64. Saleh N, Saladino G, Gervasio FL et al (2016) A Three-Site Mechanism for Agonist/Antagonist Selective Binding to Vasopressin Receptors. Angew Chem Int Ed Engl 55:8008-8012. https://doi.org/10.1002/anie.201602729

65. Crombie AL, Antrilli TM, Campbell BA et al (2010) Synthesis and evaluation of azabicyclo[3.2.1]octane derivatives as potent mixed vasopressin antagonists. Bioorg Med Chem Lett 20:3742-3745. https://doi.org/10.1016/j.bmcl.2010.04.068

66. Yamamura Y, Nakamura S, Itoh S et al (1998) OPC-41061, a highly potent human vasopressin V2receptor antagonist: pharmacological profile and aquaretic effect by single and multiple oral dosing in rats. J Pharmacol Exp Ther 287:860-867

67. Mouillac B, Chini B, Balestre MN et al (1995) The binding site of neuropeptide vasopressin V1a receptor. Evidence for a major localization within transmembrane regions. J Biol Chem 270:2577125777. https://doi.org/10.1074/jbc.270.43.25771

68. Albertazzi E, Zanchetta D, Barbier P et al (2000) Nephrogenic Diabetes Insipidus: Functional Analysis of New AVPR2 Mutations Identified in Italian Families. J Am Soc Nephrol 11:1033-1043

69. Pasel K, Schulz A, Timmermann K et al (2000) Functional characterization of the molecular defects causing nephrogenic diabetes insipidus in eight families. J Clin Endocrinol Metab 85:1703-1710. https://doi.org/10.1210/jcem.85.4.6507

70. Makita N, Manaka K, Sato J, liri T (2020) V2 vasopressin receptor mutations. Vitam Horm 113:7999. https://doi.org/10.1016/bs.vh.2019.08.012

71. Cotte N, Balestre M-N, Aumelas A et al (2000) Conserved aromatic residues in the transmembrane region $\mathrm{VI}$ of the V1a vasopressin receptor differentiate agonist vs. antagonist ligand binding. Eur $\mathrm{J}$ Biochem 267:4253-4263. https://doi.org/10.1046/j.1432-1033.2000.01472.x

72. Neocleous V, Skordis N, Shammas $C$ et al (2012) Identification and characterization of a novel Xlinked AVPR2 mutation causing partial nephrogenic diabetes insipidus: A case report and review of the literature. Metabolism 61:922-930. https://doi.org/10.1016/j.metabol.2012.01.005

73. Sahakitrungruang T, Tee MK, Rattanachartnarong N et al (2010) Functional characterization of vasopressin receptor 2 mutations causing partial and complete congenital nephrogenic diabetes insipidus in Thai families. Horm Res Pædiatrics 73:349-354. https://doi.org/10.1159/000308167

\section{Figures}




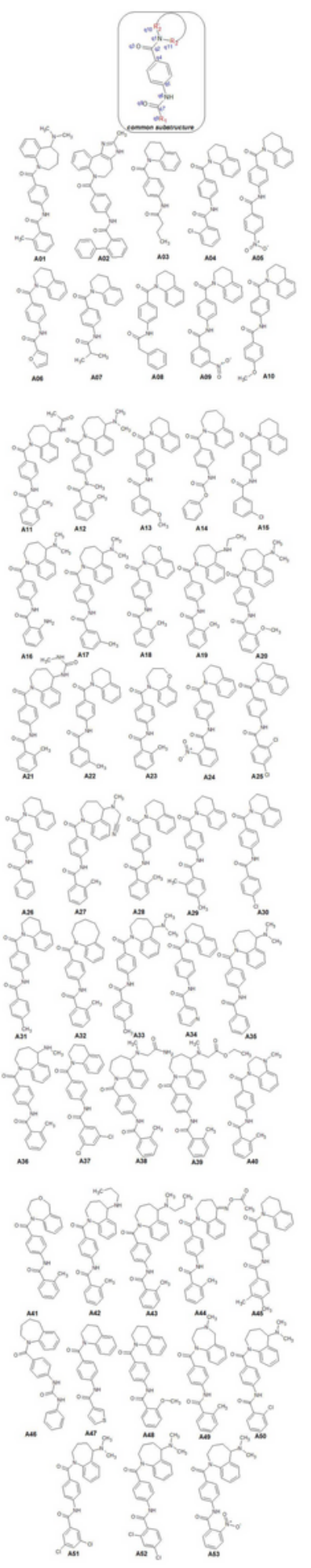

\section{Figure 1}

The common substruture (4-formamidobenzamide) of all the compounds are represented in the upper rectangle. R1 represents a variable substituent, most cases are an aromatic ring but also can be a lineal aliphatic chain. The nitrogen of the benzamide in the common substructure (represent the partial charge q1) is part of a benzazepine, benzene-piperidine or benzoxazine condensed ring. R2 and R'2 represent the 
carbon atoms next to the nitrogen part of the condensed ring. q1 to q11 represent the partial charge calculated as descriptors in this study. A1 to A53 represent all the compounds used in the study.

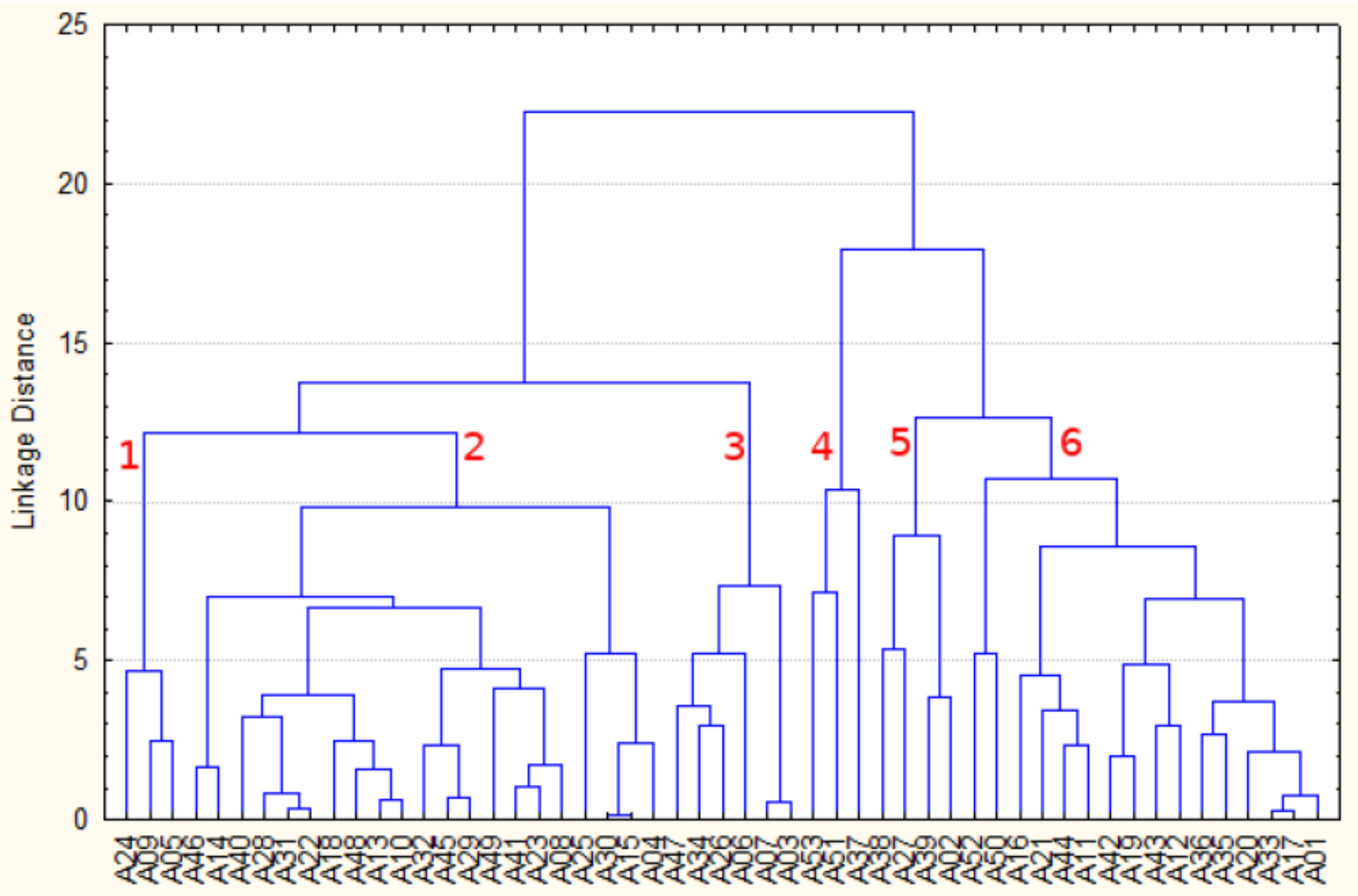

Figure 2

Dendrogram illustrating the results of the hierarchical k-NNCA of the set of 53 antagonists of V2R. The complete linkage distance was used as the connection function to merge the objects into clusters. The red numbers represent the six clusters identified. 


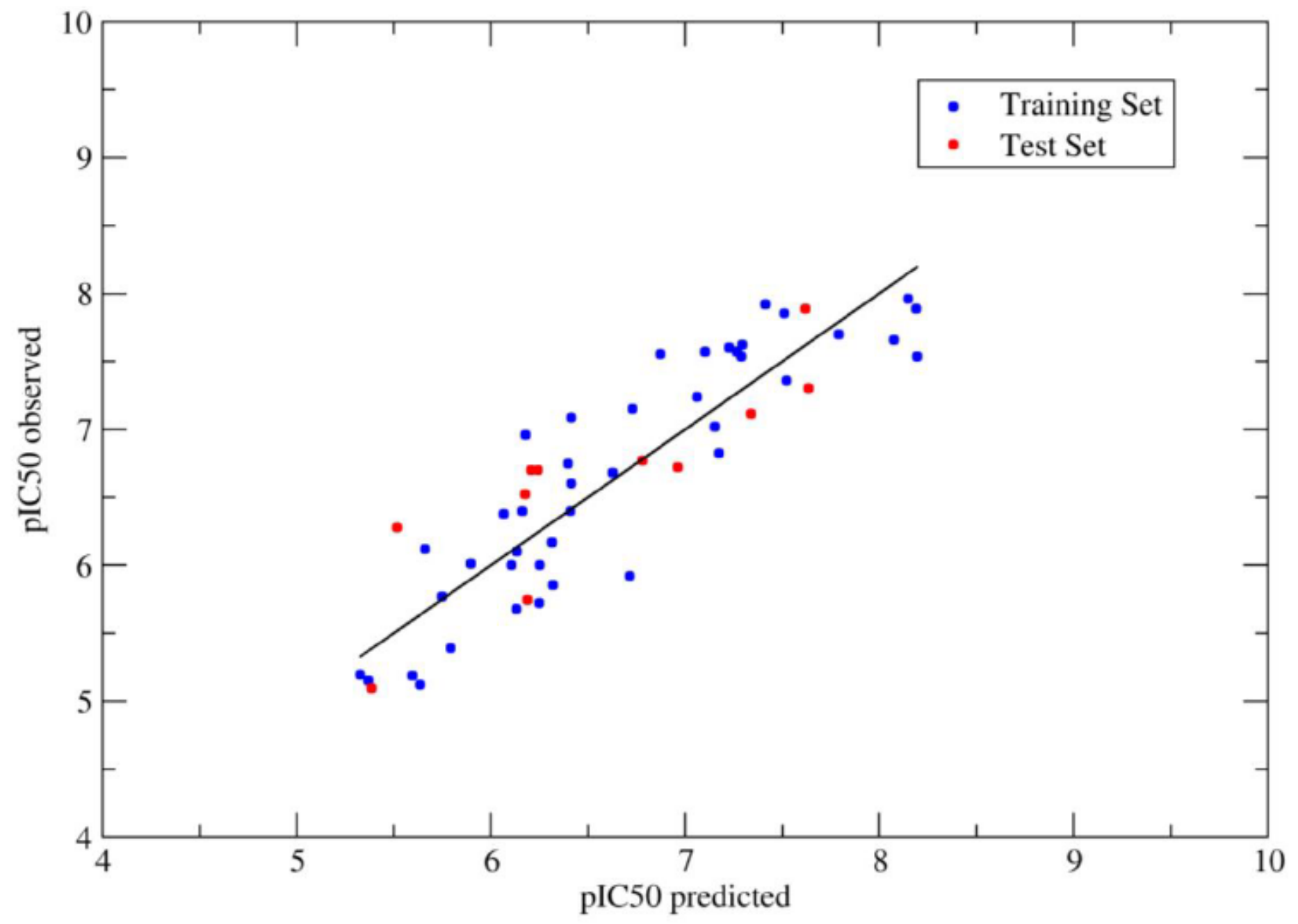

Figure 3

Observed pIC50 versus predicted pIC50. Blue and red points represent the compounds of the training and test series, respectively, and black line is the trend line. 


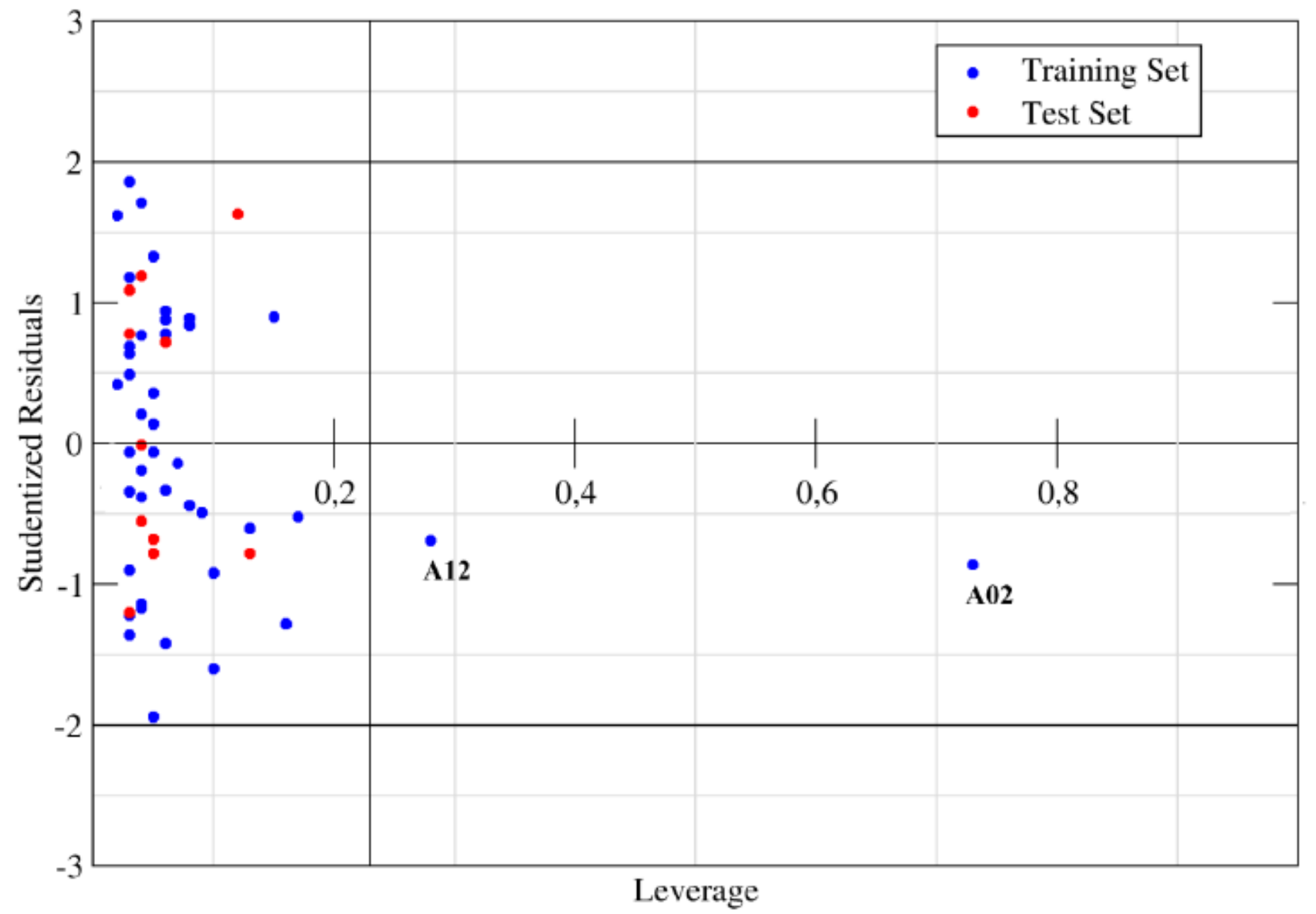

Figure 4

Williams plot (applicability domain) of Eq. (5). Blue and red points represent the compounds of the training and test series, respectively. Outlier compounds are points with standardized residual greater than two standard deviation unit. Influential chemical is a point with high leverage values higher than the threshold or cut-off value $h=0.23$. The compounds $A 02$ and $A 12$ are points with leverage greater than threshold.

V2R

$4 S O V$

V2R

4SOV

V2R

4SOV

V $2 R$

$4 S O V$

MLMASTTSAVPGHPSLPSLPSNSSQERPLDTRDPLLARAELALLSIVFVAVALSNGLVLAALARRGRRGHWAPIHVFIGHLCLADLAVALFQVLPQLAWKATDRFRGPDALCRAVKYLQM - PKEYEWVLIAGYIIVFVVALIGNVLVVAVWKNHHM . . . - VTNYFIVNLSLADVLVTITCLPATLVVDITETWFFGOSLCKVIPYLOT

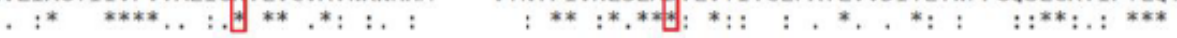

VGMYASSYMILAMTLRHRAICRPMLAYRHGSGAHWNRPVLVAWAFSLLLSLPQLFIFAQRNVEGGSGVTDCWACFAEPWGRRTYVTWIALMVFVAPTLGIAACQVLIFREIHASLVPGP VSVSVSVLTLSCIAL PRWYYAICHP . . . . . S STAKRARNSIVI IW IVSCIIMIPQAIVMECSTVF . . . FTVCDERWGGEIYPKMYHICFFLVTYMAPICLMVLAYLQIFRKLWCRQ . . .

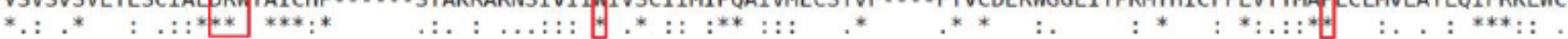

SERPGGRRRGRRTGSPGEGAHVSAAVAKTVRMTLVIVVVYVLCWAPFFLVQLWAAWDPEAPLEGAPFVLLMLLASLNSCTNPWIYASFSSSVSSELRSLLCCARGRTPPSLGPQDESCTT

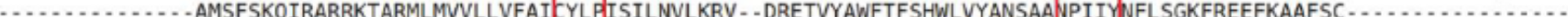

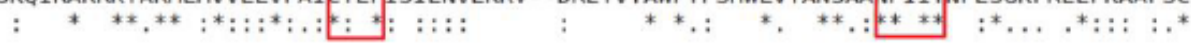

ASSSLAKDTSS

A............ 


\section{Figure 5}

Sequence alignment used for modeling V2R. The blue cylinders mark the transmembrane helices. In red boxes are the conserved motifs of each transmembrane helix.
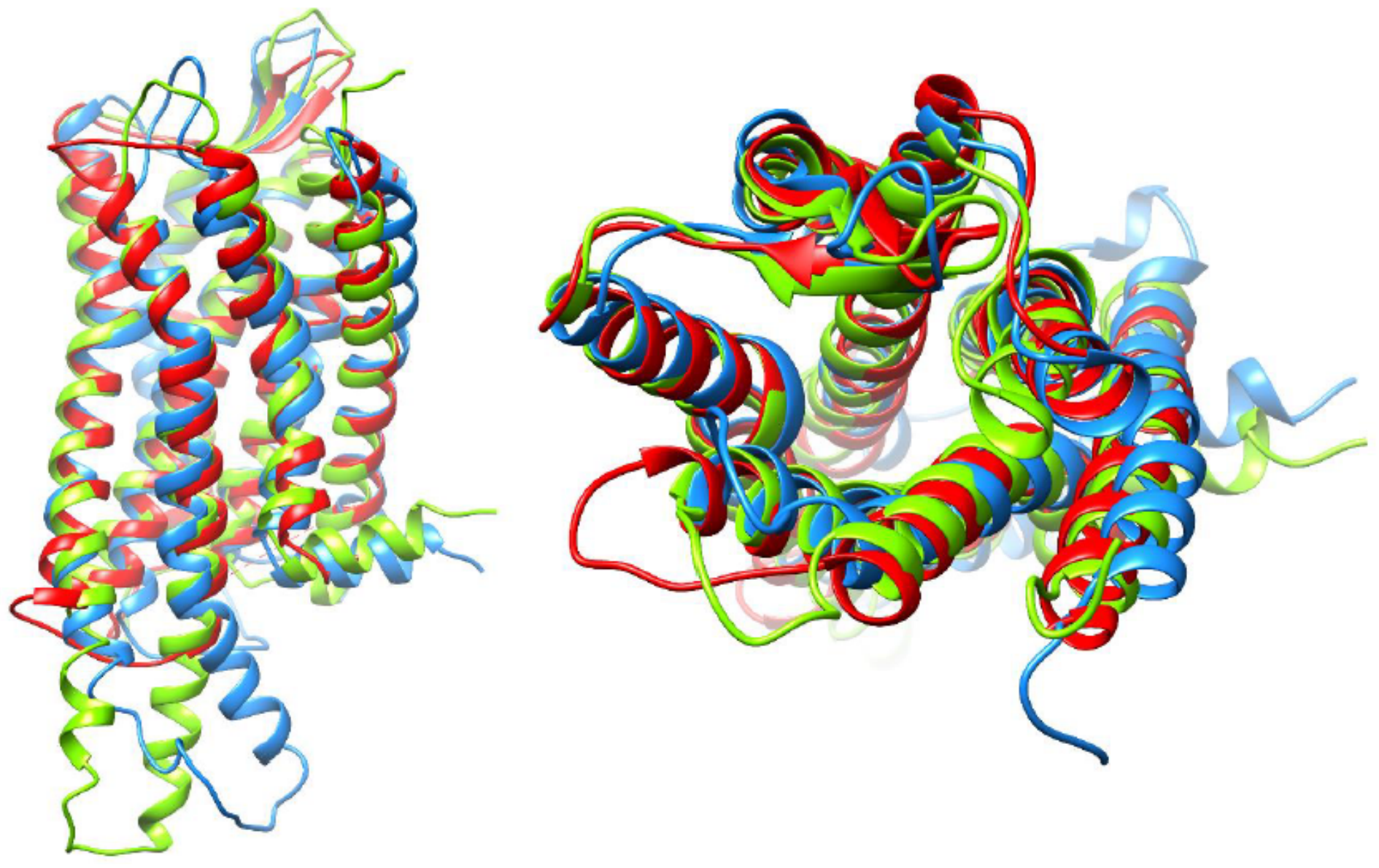

Figure 6

Models superposition on oxytocin receptor (OXTR). In red $x$ ray structure of OXTR (PDB_ID 6TPK), in green V2R model (template 4SOV), in blue V2R model minimized and relaxed in POPC membrane. 


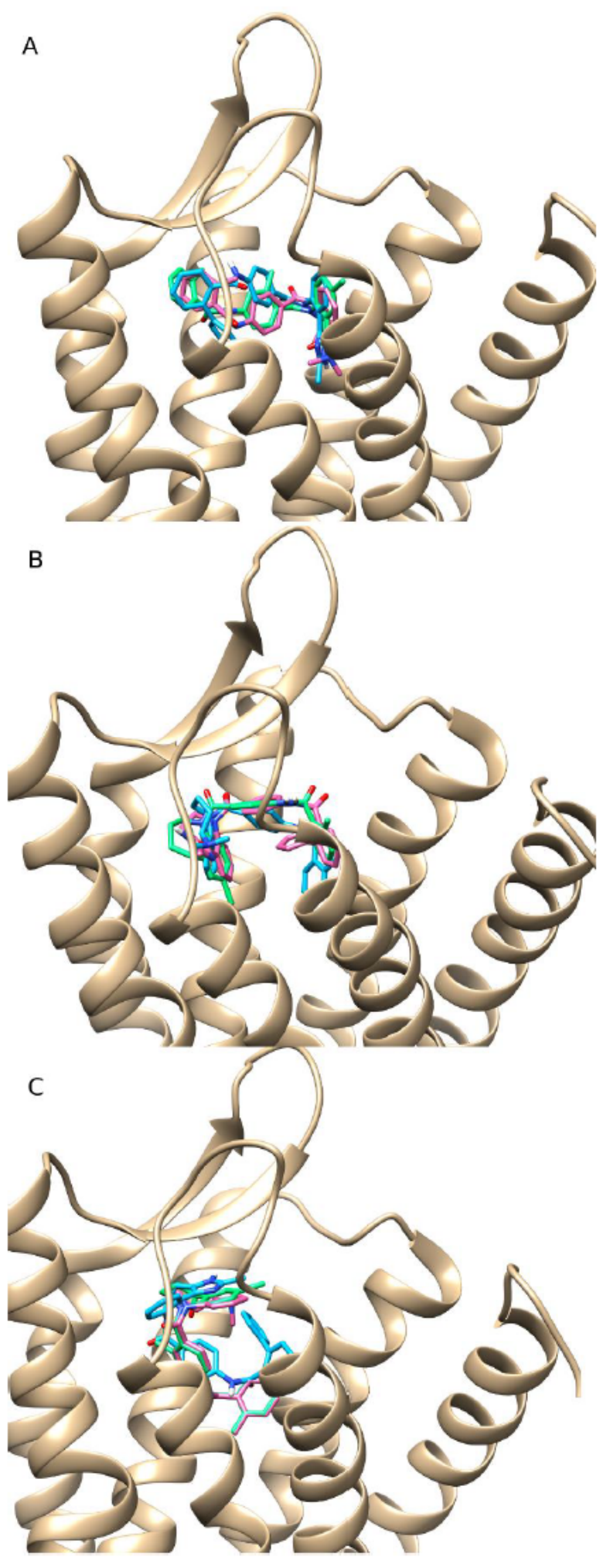

Figure 7

Representative V2R-antagonist complexes obtained by molecular docking. The antagonists are represented in different color: tolvaptan (green), mozavaptan (pink), conivaptan (blue). A) conformation with condensed ring toward TM 2 and 7 (CR-27). B) conformation with condensed ring toward TM 5 and 6 (CR-56). C) conformation with condensed ring at the top of the binding site (CR-UP) 


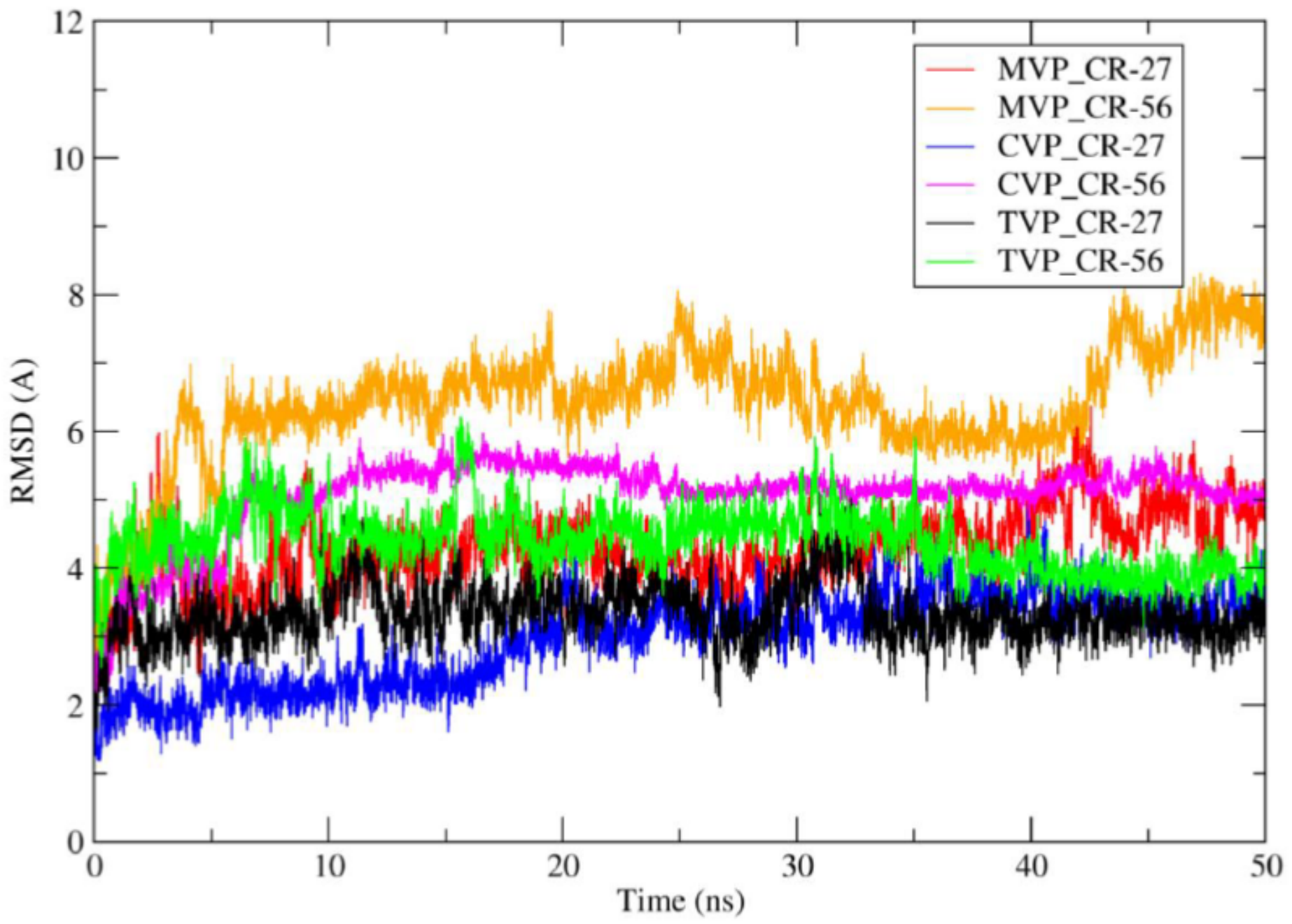

Figure 8

Root mean square deviation (RMSD) of the antagonists during the molecular dynamic simulation. MVP: mozavaptan, CVP: conivaptan and TVP: tolvaptan. CR-27: conformation with condensed ring toward TM 2 and 7 . CR-56 conformation with condensed ring toward TM 5 and 6. 

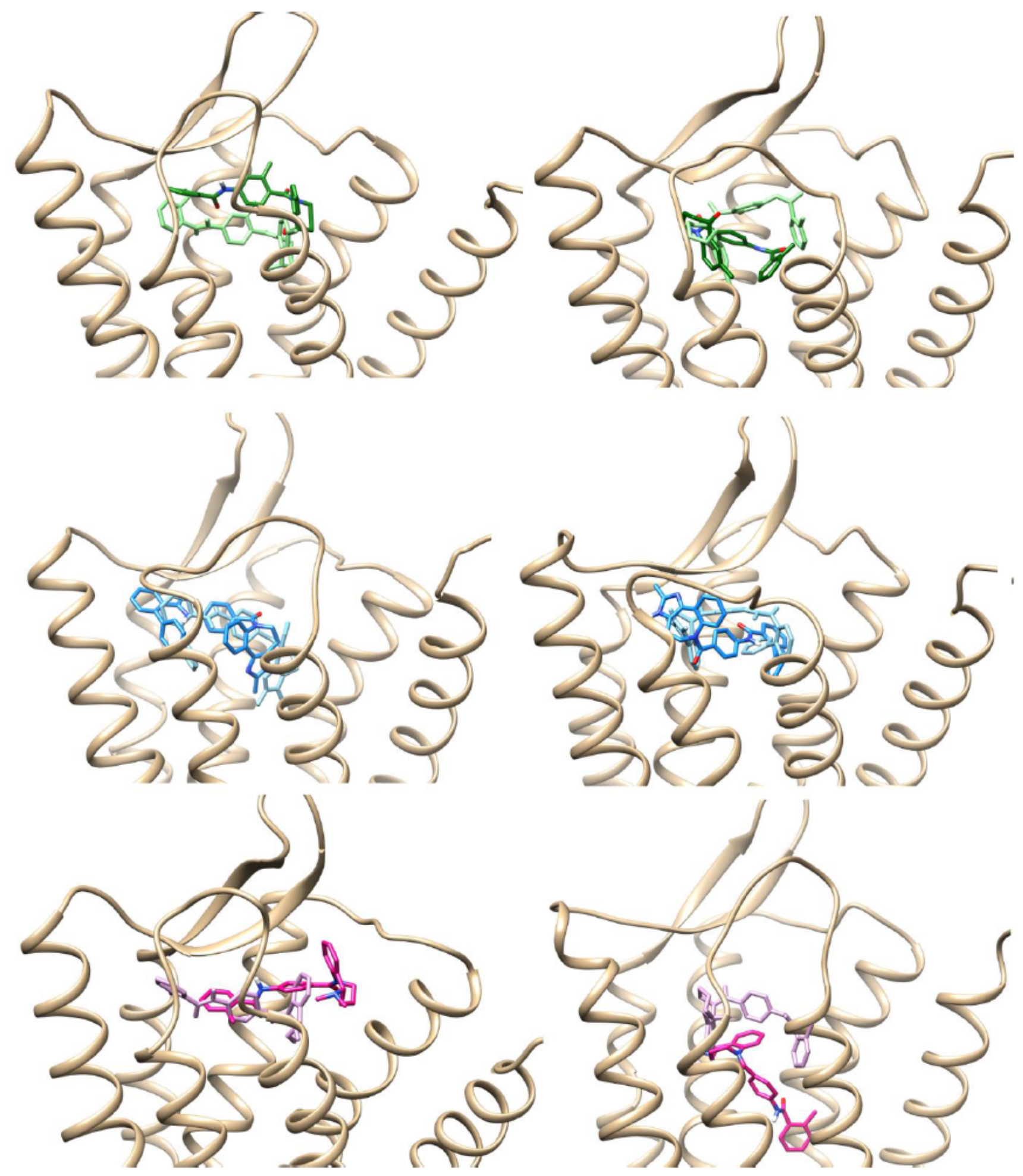

Figure 9

Complexes studied by molecular dynamics simulations. The upper line shows the two representative tolvaptan (green) complexes, the middle line shows conivaptan (blue) complexes, the bottom line shows mozavaptan (pink) complexes. From left to right are represented the conformation CR-27 and CR-56 respectively. For each complex, the light ligand represents the conformation at time Ins and the dark ligand represents the conformation at time 50ns.

Page $40 / 42$ 


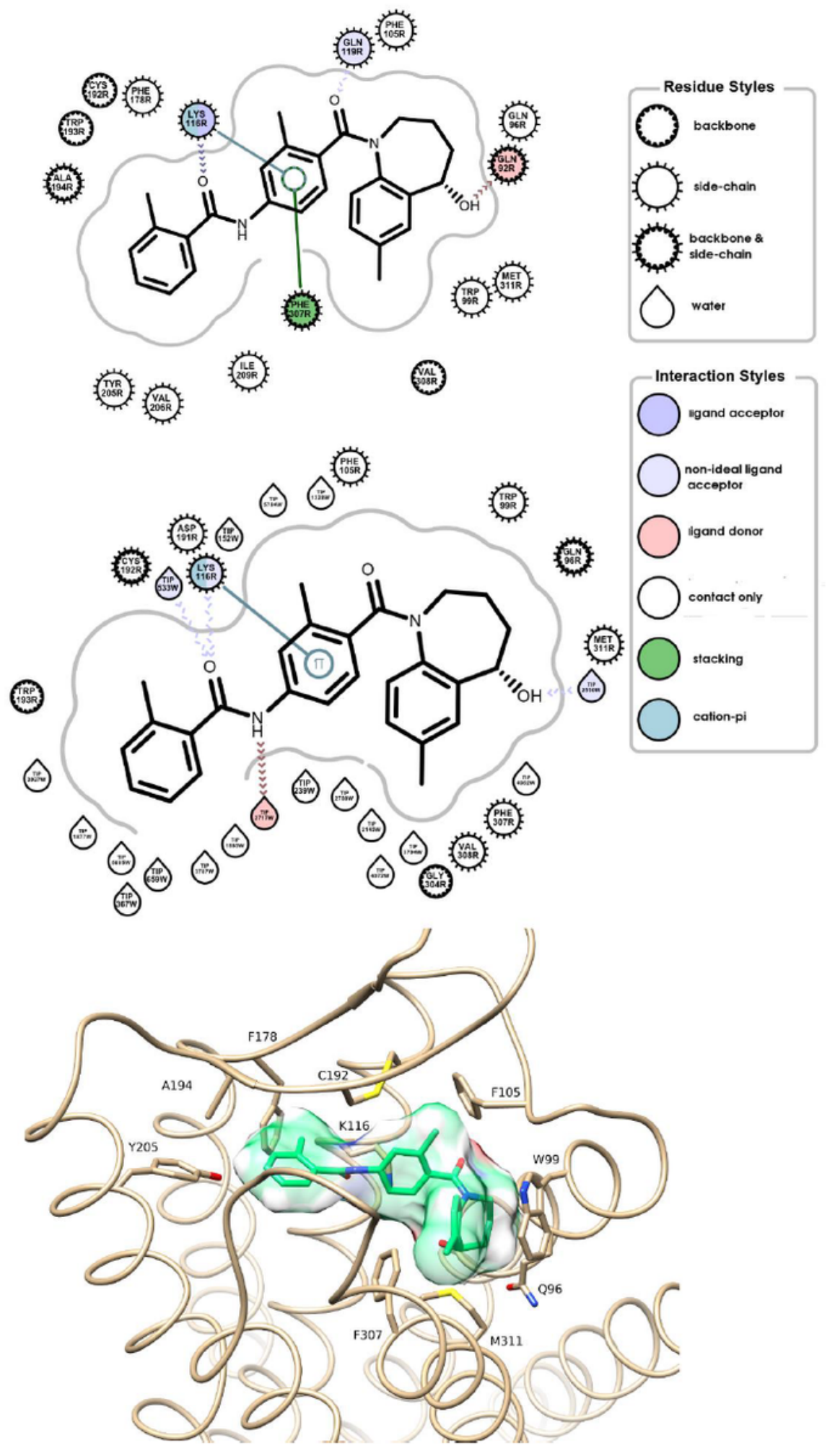

Figure 10

Tolvaptan -V2R complex (CR27 conformation). Top panel shows interaction map before molecular dynamic simulation. Middle panel shows interaction map at 50ns of molecular dynamic simulation. Bottom panel shows 3D representation of the complex at $50 \mathrm{~ns}$ of molecular dynamic simulation.

\section{Supplementary Files}


This is a list of supplementary files associated with this preprint. Click to download.

- delaNuezV2RSupplementaryInfomationTables.xlsx

- delaNuezV2RSupplementaryInformationFigures.pdf 